\title{
Aging changes in the retina of male albino rat: a histological, ultrastructural and immunohistochemical study
}

\author{
M.E.I. Mohamed, E.A.A. El-Shaarawy, M.F. Youakim, D.M.A. Shuaib, M.M. Ahmed \\ Department of Anatomy and Embryology, Faculty of Medicine, Cairo University, Cairo, Egypt \\ [Received: 9 June 2018; Accepted: 26 June 2018]
}

Background: Degenerative changes caused by aging may affect the eye, especially the retina. Such changes occur as a part of normal physiological process and may be irreversible. The aim of the study was to demonstrate the influence of aging on the morphology of the retina to provide a basis to explain the pathogenesis of age-associated decline in visual acuity, scotopic and photopic sensitivity.

Materials and methods: Forty male albino rats were used and divided into four age groups (group I: age of cortical maturity, group II: middle-aged, group III: aged group and group IV: senile group). The rats were sacrificed, the eye balls were enucleated. Intra-vitreal injections of formalin for haematoxylin and eosin and immunohistochemical sections, glutaraldehyde for toluidine blue semithin and E/M ultra-thin sections were performed. Measurements and quantitative histomorphometric estimation of the layers of the retina were done.

Results: Light microscopic examination revealed age-dependent attenuation of photoreceptor striations. Aged and senile groups presented pyknotic, widely-spaced nuclei of the outer nuclear layer. The inner nuclear layer was thinned out to 2 or 3 cellular rows. Retinal capillaries showed progressive dilatation and congestion. Statistical analysis proved significant thinning of the retina with variable degrees of thinning of the constituting layers. Decreased arborisation with age was confirmed with quantification of synaptophysin-immunostained sections. Glial fibrillary acidic protein immunostaining revealed the picture of reactive gliosis. On the ultrastructural level, the retinal pigment epithelium exhibited major alterations with aging. Numerous phagosomes, lipofuscin and melanolipofusin granules appeared within the cells, together with exaggerated basal infoldings. The photoreceptor nuclei became degenerated and the perinuclear space was widened. Conclusions: Rat retinae clearly undergo age-related morphological changes. Such changes provide a cellular base for explanation of decreased vision in humans with aging other than reflection errors. Effect of aging was not only qualitative, but also quantitative. (Folia Morphol 2019; 78, 2: 237-258)

Key words: retina, ageing, ultrastructure, immunohistochemical, histology 


\section{INTRODUCTION}

The development of the visual system involves a sequence of neurochemical signals which regulate synaptic connections and those processes modulate the visual experience [23]. Older people may experience alteration of vision, even in the absence of any identifiable eye disease. Advancing age has been associated with decrease in visual acuity and reduction in scotopic sensitivity, contrast sensitivity and motion sensitivity as well as poor performance on different visual discrimination tasks [19].

The vertebrate retina, like other parts of the central nervous system, is subjected to degenerative changes caused by aging. In addition, the retina is a site of diseases for which age is a major risk factor. The retina is the best understood part of the vertebrate central nervous system with regard to its cellular patterning, circuitry and function [32]. The retina is composed of five major neuron types: retinal ganglion cells, amacrine cells, bipolar cells, horizontal cells and photoreceptors that integrate visual information and send it to the brain [32]. The neuronal somas are located in three nuclear layers whose axonal and dendritic processes form complex and orderly networks of chemical and electrical synapses forming two plexiform layers; outer and inner. Synaptophysin is an integral membrane protein of the synaptic vesicles. It possibly serves multiple functions in synaptic vesicle formation and exocytosis, playing an important role in neurotransmitter delivery. It is widely used as one of the synaptic function markers. The expression pattern of synaptophysin in retina indicates an important role in ribbon synapse formation and visual signal transmission [11]. Retinal macroglia (MG), consisting of astrocytes and Müller cells, play key roles in the homeostasis of retinal neurons, keep the retina healthy and functioning properly. Mammalian MG are quiescent and respond to retinal damage by reactive gliosis, rarely with proliferation, and do not naturally regenerate neurons [18].

Astrocytes, almost entirely restricted to the retinal nerve fibre layer, have close relationship with neurons and the major blood vessels. They are commonly thought to play an important part in the proper development and functioning of the vascular system in the retina, including blood flow and formation of the blood-retinal barrier [21]. Astrocyte activation and reactive gliosis are common traits in neurodegenerative processes. A hallmark of gliosis is the upregulation of intermediate filament proteins, including glial fibril- lary acidic protein (GFAP) in glial cells [9]. The aim of the present study was to determine the histological and immunohistochemical changes in the retina of male albino rats that occur with aging and demonstrate the ultrastructural age-related alterations and their correlation with the histological changes.

\section{MATERIALS AND METHODS}

\section{Animals}

The present study was carried out on 40 male albino rats. The rats were selected to fall equally into four age groups, chosen in correspondence to human [33] as follows:

- group I: 6 months old (age of cortical maturity);

— group II: 12 months old (middle-aged);

- group III: 24 months old (aged);

- group IV: 30 months old (senile).

The rats were obtained from the Animal House, Research Institute of Ophthalmology. They were housed in cages, 5 rats/cage, under standard laboratory and environmental conditions 2 weeks before selection. The rats were sacrificed by cervical dislocation and the eye balls were enucleated. The upper nasal part of the sclera was pierced $1.5 \mathrm{~mm}$ posterior to the limbus, with a 30 gauge Hamilton needle fitted to a syringe. The needle tip was visualised during the procedure to avoid retinal injury, with intraocular injections in the posterior vitreous of the eye behind the lens [38] either with formalin for haematoxylin and eosin (H\&E) and immunohistochemical sections or with glutaraldehyde for toluidine blue stain semithin and E/M ultrathin sections.

\section{Methodology}

Light microscopy. The right eye balls were injected with $10 \%$ formalin in the posterior chamber of the eye behind the lens and left for $24 \mathrm{~h}$. The peripheral retinae were obtained and processed.

Histological examination using H\&E stain.

Immunohistochemical staining done for:

- GFAP: was performed to detect glial cell reactivity [8]. Positive control (brown discoloration of the glial cells mainly astrocytes denoting increase gliosis) was IMR5 cells in brain. For negative controls, incubation was carried out with the omission of the primary antiserum.

- Synaptophysin (SYN) was applied, to detect synaptic functions [2]. SYN is a synaptic vesicle glycoprotein. It is present in almost all neuroendocrine cells that participate in synaptic transmission. 


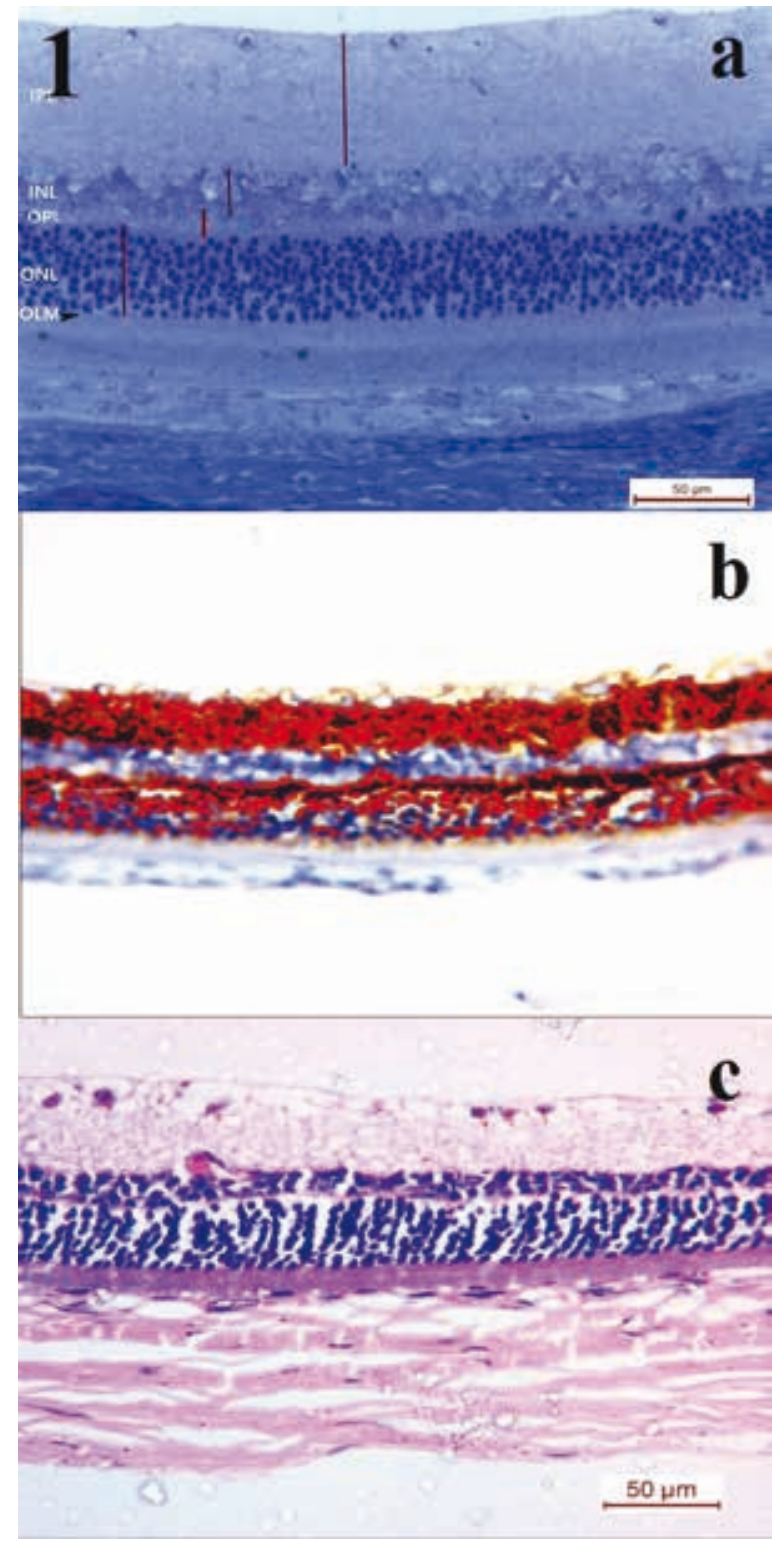

Figure 1. An image displayed on the monitor screen of a field in rat retina showing: a. Measuring of the thickness of different layers; b. Measuring of synaptophysin optical density; c. Ganglion cell counting; OLM — outer limiting membrane; ONL — outer nuclear layer; OPL — outer plexiform layer; INL — inner nuclear layer; IPL — inner plexiform layer.

Its ubiquity at the synapse has led to the use of SYN immunostaining for the quantification of synapses [36].

Electron microscopy. The retinae of the left eye balls were prepared for ultrastructural sections for examination using transmission electron microscope. Parts of the peripheral retinae were obtained and processed. The sections were examined and photographed by JEOL JEM 1400 transmission electron microscope in the Electron Microscope Research Laboratory, Faculty of Agriculture Research Centre.

Morphometric study. Image J image analysis software was used to analyse the photomicrographs of the different groups taken at magnification $\times 400$ using Leica ICC50 microscope equipped with digital camera. The following measurements were done (Figs. 1a-c):

- The thickness of the retina, inner plexiform layer (IPL), inner nuclear layer (INL), outer plexiform layer (OPL) and outer nuclear layer (ONL) thickness: to quantify the thickness of the retina and its nuclear and synaptic layers, images were taken at equivalent retinal eccentricities from the optic nerve head. Layer thickness was measured in two to four areas from each retina. The measurements were done using semithin epoxy resin sections photomicrographs.

- Measurement of the density of synapses: using the photomicrographs of SYN-immunostained sections.

- Measurement of the ganglion cell count $/ 300 \mu \mathrm{m}$ ganglion cell layer (GCL): using the photomicrographs of H\&E sections.

\section{Statistical analysis}

Statistical measures were done using SPSS programme, version 20. One way analysis of variance (ANOVA) was employed to compare means between groups. Bonferroni post hoc test was used to detect significance between every two individual groups. The mean, standard deviation and the $p$ value were calculated for each experimental group and compared among the different groups. Significance was considered when $p$-value was $\leq 0.05$. The data were examined by the Kolmogorov-Smirnov test for normality.

\section{RESULTS}

\section{Light microscopic results}

Group I (cortical maturity). The retina of group I featured the 10 layers, from outside inwards: retinal pigment epithelium (RPE), photoreceptor layer of rods and cones with outer lightly stained segment and inner deeply stained segment, outer limiting membrane (OLM), outer nuclear layer, outer plexiform layer, inner nuclear layer, inner plexiform layer, ganglion cell layer, nerve fibre layer and inner limiting membrane (ILM) (Fig. 2a, b). The RPE appeared as a single layer of cuboidal cells with ill-defined boundaries and pale, oval, vesicular nuclei. This layer was lying on Bruch's 


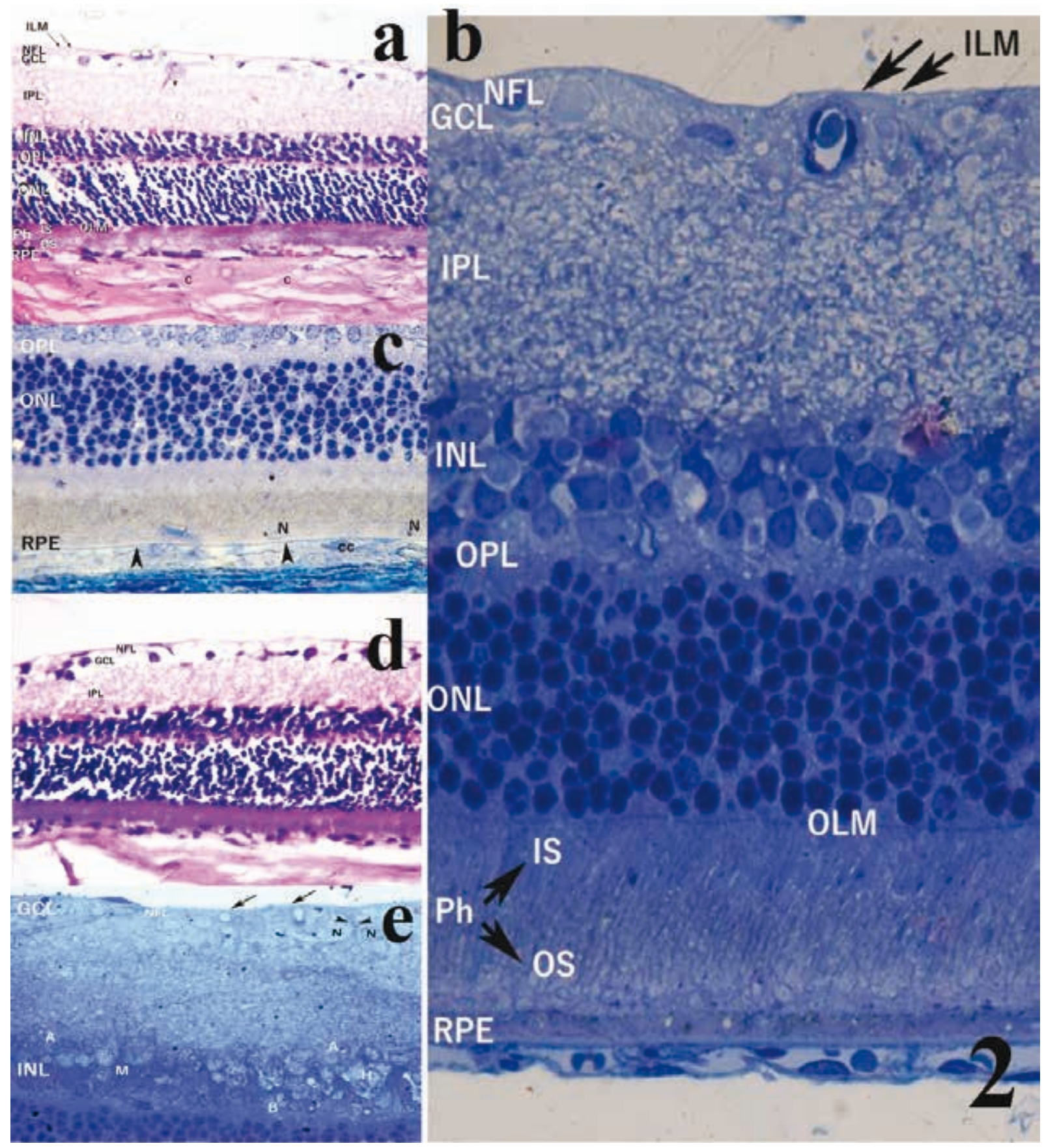

Figure 2. A micrograph of rat retina (group I) showing: a. The arrangement of the 10 layers, from choroid (C) inwards as: retinal pigment epithelium (RPE), photoreceptor layer (Ph) with outer lightly stained outer segment (OS) and inner deeply stained inner segment (IS), outer limiting membrane (OLM), outer nuclear layer (ONL), outer plexiform layer (OPL), inner nuclear layer (INL), inner plexiform layer (IPL), ganglion cell layer (GCL), nerve fibre layer (NFL) and inner limiting membrane (arrows); H\&E $\times 400$; $\boldsymbol{b}$. A semithin section with 10 layers, from outside inwards; retinal pigment epithelium (RPE), photoreceptor layer (Ph) of rods and cones with outer segment (OS) and inner segment (IS), outer limiting membrane (OLM), outer nuclear layer (ONL), outer plexiform layer (OPL), inner nuclear layer (INL), inner plexiform layer (IPL), ganglion cell layer (GCL), nerve fibre layer (NFL) and inner limiting membrane (arrows); toluidine blue $\times 1250$; c. Semithin section of rat retina (group I) showing the retinal pigment epithelium (RPE) layer with pale nuclei (N) lying on the Bruch's membrane (arrowheads). Choriocapillaries (CC) are seen beneath the Bruch's membrane. The outer nuclear layer $(\mathrm{ONL})$ is formed of multiple rows of nuclei of the photoreceptors. The outer plexiform layer (OPL) forms a dense network of synapses; toluidine blue $\times 1250$; $\mathbf{d}$. Retinal ganglion cell layer (GCL), covered with the nerve fibre layer (NFL). The inner plexiform layer (IPL) is formed of network of synapses; H\&E $\times 1000$; e. Semithin section of rat retina (group I) showing the four types of cells identified in inner nuclear layer (INL); horizontal cells $(H)$, bipolar cells $(B)$, amacrine cells $(A)$ and Müller cells (M). In ganglion cell layer (GCL), the cells exhibit pale nuclei (N) and their axons (arrowsheads) exit to form nerve fiber layer (NFL). The inner limiting mebrane (arrows) is seen; toluidine blue $\times 1250$. 


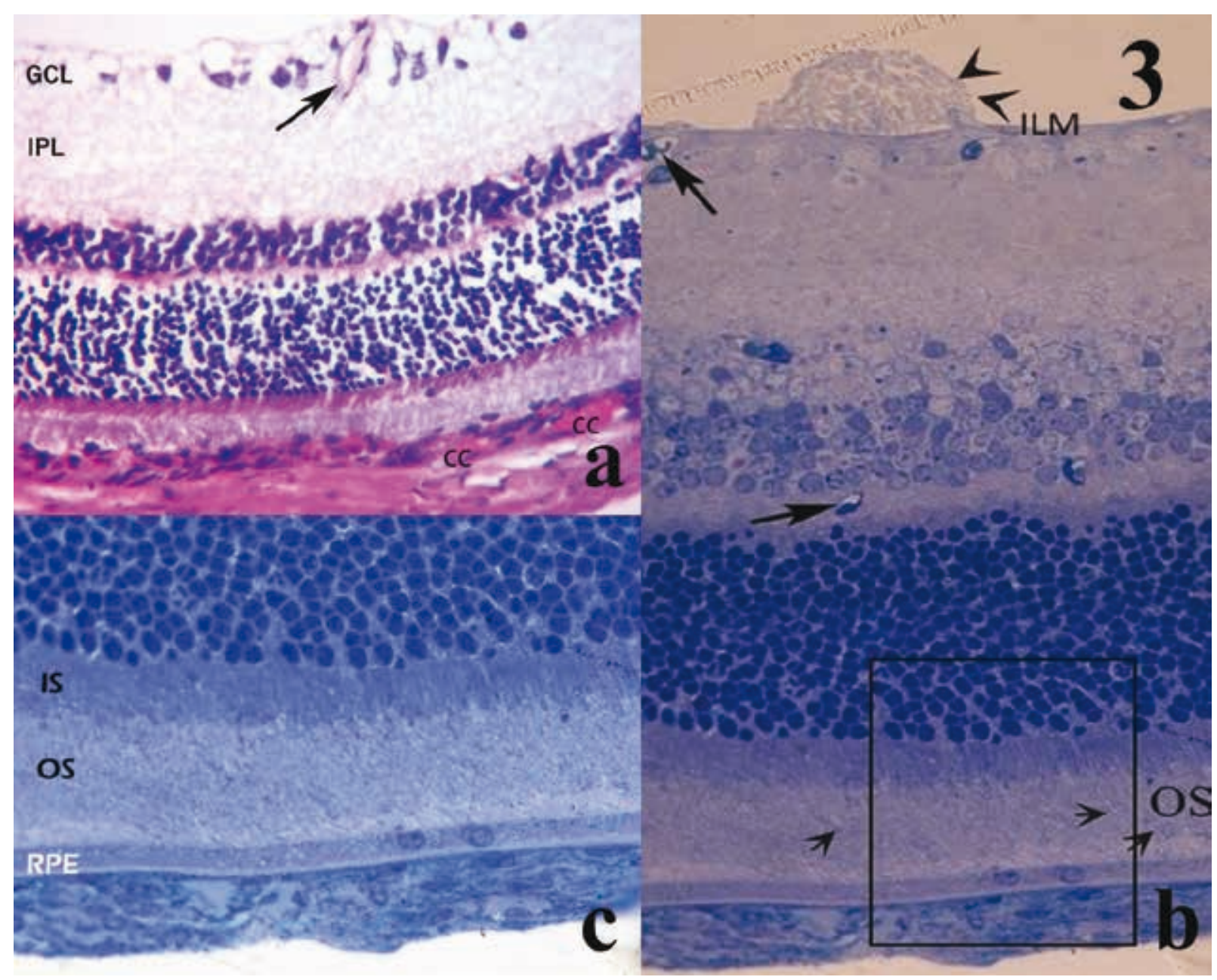

Figure 3. A micrograph of rat retina (group II) showing: a. The ganglion cell layer (GCL) exhibiting a dilated blood vessel (arrow) extending into the inner plexiform layer (IPL). The choriocapillaries (CC) are congested; H\&E $\times 400$; $\mathbf{b}$. Photoreceptors outer segments (OS) exhibiting fragmentation (short arrows). Exudate (arrowheads) on the inner limiting membrane (ILM) and dilated blood vessels (long arrows) in the outer plexiform layer are observed; toluidine blue $\times 1250$; c. A higher magnification of the inset in the previous figure revealing attenuated striations in the outer (OS) and the inner (IS) segments of photoreceptors. The retinal pigment epithelium (RPE) is shown; toluidine blue $\times 1250$.

membrane which separates it from the choroid (Fig. 2c). The photoreceptor layer appeared as fibrillary striations, composed of an outer lightly stained segment and an inner, deeply stained segment. The outer nuclear layer was formed of nuclei of photoreceptors which form multiple deeply-stained rows (Fig. 2b, c). Their axons synapsed with dendrites of inner nuclear layer cells forming the dense outer plexiform layer (Fig. 2c). A thicker network of synapses, the inner plexiform layer, is formed by arborisation of axons of the inner nuclear layer cells, together with dendrites of the ganglion cell layer (Fig. 2d). Four types of cells could be identified in the inner nuclear layer: horizontal cells with large pale nuclei, bipolar cells with smaller, rounded or oval nuclei and chromatin clumps, amacrine cells with indented nuclei and angulated Müller cells in between the other cell types (Fig. 2e). The ganglion cells was aligned as a single layer of nuclei (Fig. 2d, e). Their axons exit as the nerve fibre layer limited internally by the inner limiting membrane (Fig. 2e).

Group II (middle-aged). The rat retina of group II displayed mild dilatation of blood vessels in ganglion cell layer extending into the inner plexiform layer (Fig. 3a) and in the outer plexiform (Fig. 3b). Exudation of the inner limiting membrane was seen in rare foci of this group (Fig. 3b), photoreceptors layer showed attenuation of normal striation and mild outer segment fragmentation (Fig. 3b, c).

Group III (aged). Group III showed apparent decrease in the whole retinal thickness (Fig. 4a) especial- 


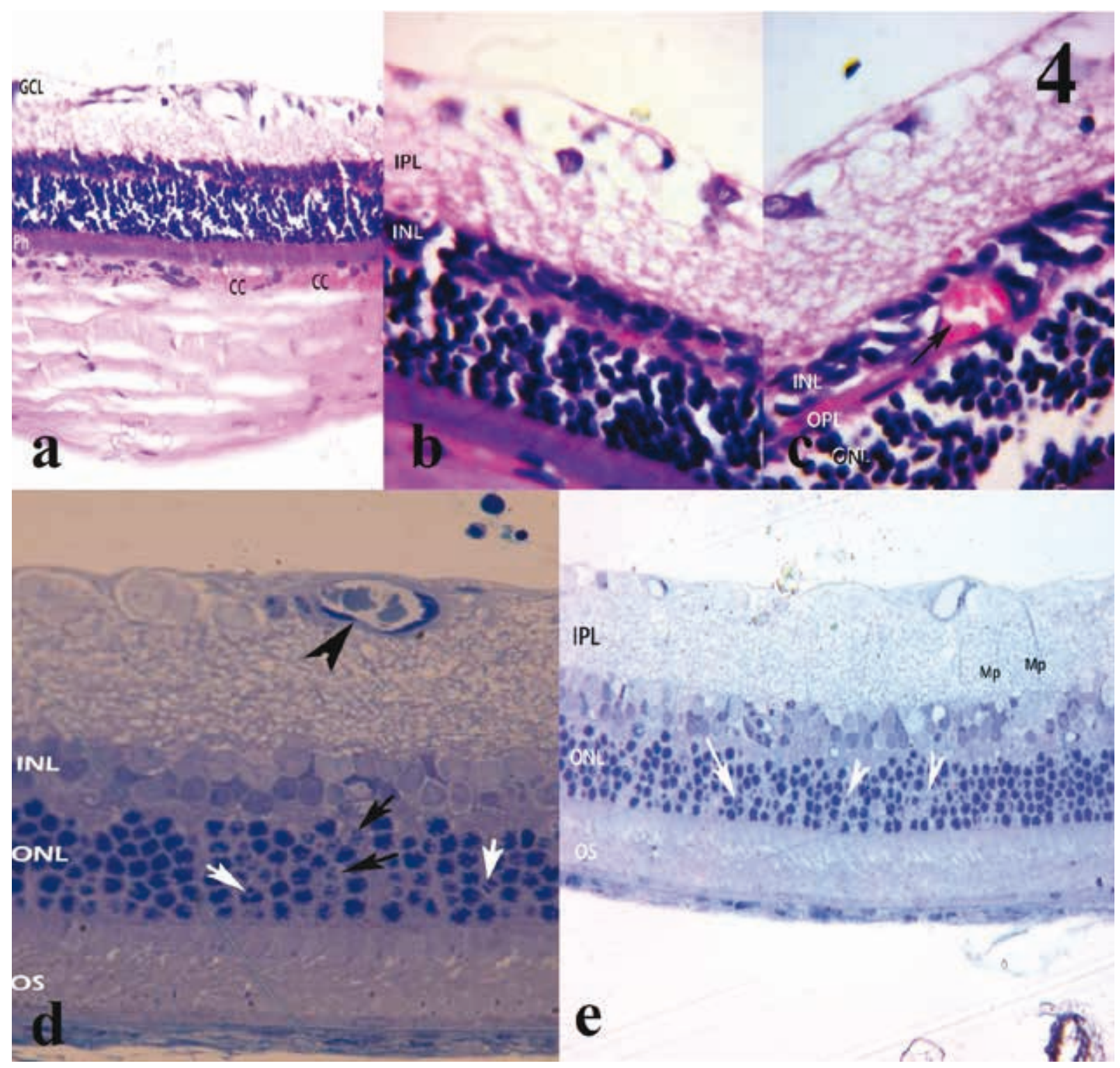

Figure 4. A micrograph of rat retina (group III) showing: a. Thinning out of the retina. A dilated blood vessel in ganglion cell layer and dilated congested choriocapillaries (CC) are observed. Cells of the ganglion cell layer (GCL) exihibit irregular distribution. The photoerceptor layer (Ph) shows attenuated striations; H\&E $\times 400$; b. Thinning of the inner plexiform layer (IPL) and the inner nuclear layer (INL); H\&E $\times 1000$; c. Dilated blood vessel (arrow) in the outer plexiform layer (OPL) which encroaches on the inner nuclear layer (INL). Spacing between cells of the outer nuclear layer (ONL) is seen; $\mathrm{H} \& \mathrm{E} \times 1000$; d. Fragmentation of photoreceptor outer segments (OS), low cell density is observed in both outer (ONL) and inner (INL) nuclear layers. Pyknotic nuclei (white arrows) and ghost cells (black arrows) ware seen. A dilated blood vessel with thickened wall (arrowhead) in ganglion cell layer is also seen; toluidine blue $\times 1250$; e. Loss of striations in photoreceptor outer segments (OS). Dark irregular pyknotic nuclei (arrows) and ghost cells (arrowheads) are seen in the outer nuclear layer (ONL). Müller cell processes (Mp) are observed in inner plexiform layer (IPL); toluidine blue $\times 1000$.

ly of the inner plexiform and the inner nuclear layers (Fig. 4b). The photoreceptor outer segments showed attenuated striations (Fig. 4a) while the outer nuclear layer showed spacing between the photoreceptor nuclei (Fig. 4c). Semithin sections showed fragmentation of the photoreceptor outer segments (Fig. 4d). Some photoreceptor nuclei appeared pyknotic; moreover, ghost cells were encountered (Fig. 4d, e).
The inner retina was thinned out and the inner nuclear layer was formed of two to three cellular rows (Fig. 4b-d). The ganglion cells displayed irregular distribution along the ganglion cell layer (Fig. 4a). Dilatation of retinal blood vessels was observed (Fig. 4a, C, d) with thickening of their wall (Fig. 4d).

Group IV (senile). The rat retina of group IV presented thinning of different layers (Fig. 5a) and 


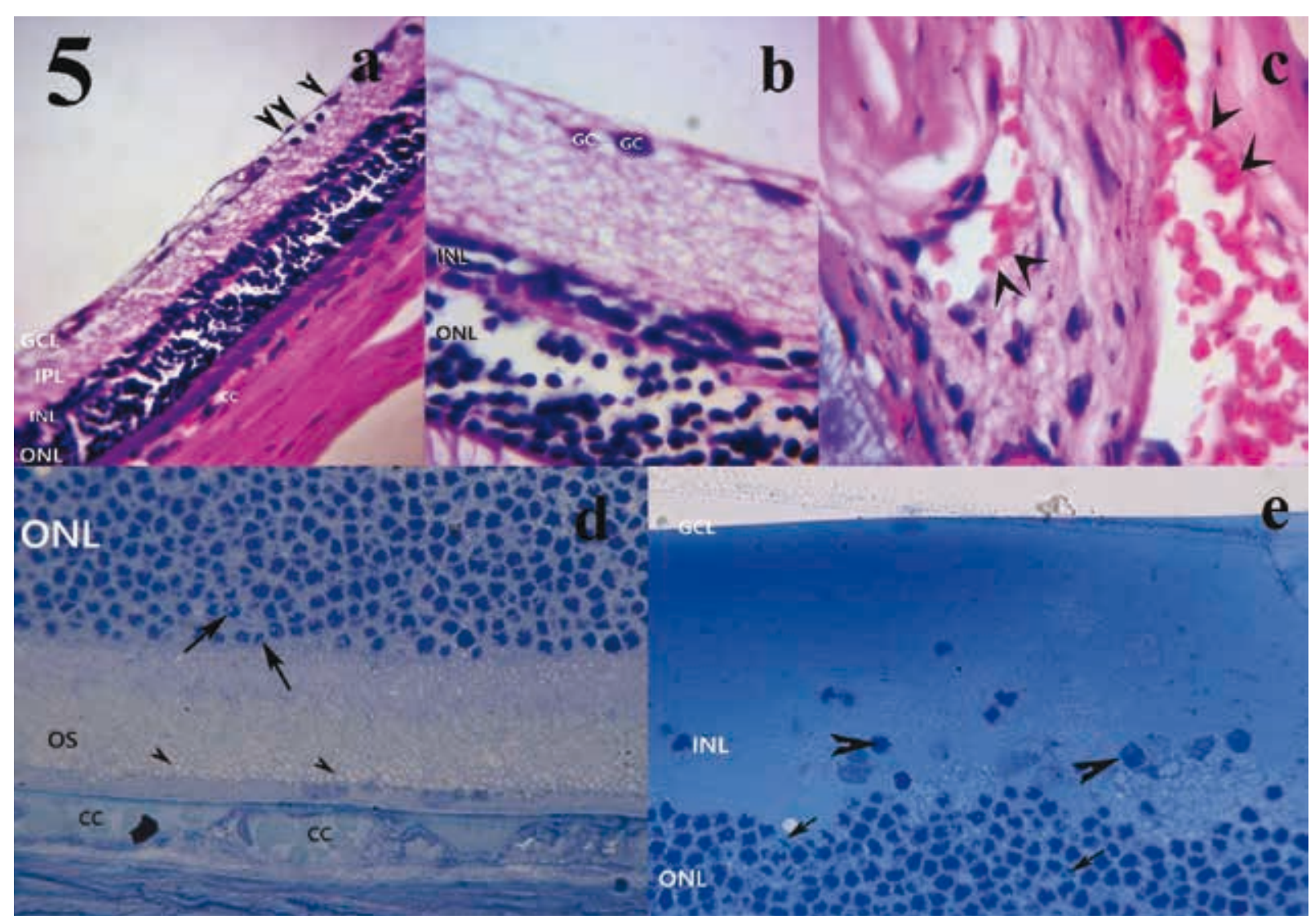

Figure 5. A micrograph of rat retina (group IV) showing: a. Thinning out of the retina particularly the inner nuclear layer (INL) and the inner plexiform layer (IPL). Congestion of choriocapillaries (CC), spacing between photoreceptor nuclei in the outer nuclear layer (ONL) and irregular distribution of cells (arrowheads) in the ganglion cell layer (GCL) are seen; H\&E $\times 400 ; \mathbf{b}$. Two to three cellular rows forming the inner nuclear layer (INL) and marked spacing between outer nuclear layer (ONL) cells. Ganglion cells (GC) are irregularly distributed; H\&E $\times 1000$; c. Areas of severe dilatation and congestion of choriocapillaries (arrowheads); H\&E $\times 1000$; d. Dilated congested choriocapillaries (CC), foamy appearance (arrowheads) of photoreceptor outer segments (OS) and degenerating nuclei (arrows) in the outer nuclear layer (ONL); toluidine blue $\times 1250$; e. Degenerating photoreceptor nuclei (arrows) in the outer nuclear layer (ONL), severely affected inner nuclear layer (INL) cells (arrowheads) and rarity of cells in the ganglion cell layer (GCL); toluidine blue $\times 1250$.

the inner nuclear layer was formed only of two to three cellular rows (Fig. 5b). Choriocapillaries were markedly dilated and congested (Fig. 5s, c, d). The photoreceptor layer exhibited a foamy appearance (Fig. 5d). The outer nuclear layer showed spacing between cells (Fig. 5a, b) and contained many irregular, degenerating nuclei (Fig. 5d, e). The inner nuclear layer displayed severe damage in some specimens (Fig. 5e). The ganglion cells exhibited irregular distribution throughout this layer (Fig. 5a, b) with rarity of cells in some specimens (Fig. 5e).

Regarding the GFAP staining of rat retina in control group I showed positive immune reaction which is confined to the nerve fibre layer (Fig. 6a). SYN staining revealed strong positive immune reaction in the two synaptic layers, IPL and OPL (Fig. 6b).
Group II sections showed positive immune reaction for GFAP labelling the nerve fibre layer and Müller cell processes extending into the inner portion of IPL (Fig. 6c). SYN showed strong positive reaction in the IPL and OPL (Fig. 6d). GFAP immunostaining revealed strong positive reaction (in group III) at the nerve fibre layer together with staining of Müller cells processes throughout the retinal layers (Fig. 6e). SYN showed moderate reaction in the inner and outer plexiform layers (Fig. 6f). In group IV stained with GFAP immunostaining, numerous Müller cell processes were stained positively and seen traversing the inner and outer plexiform layers; along with a strong positive reaction of astrocytes at the nerve fibre layer (Fig. $6 \mathrm{~g}$ ). The inner and outer plexiform layers gave a weak positive reaction to synaptophysin (Fig. 6h). 


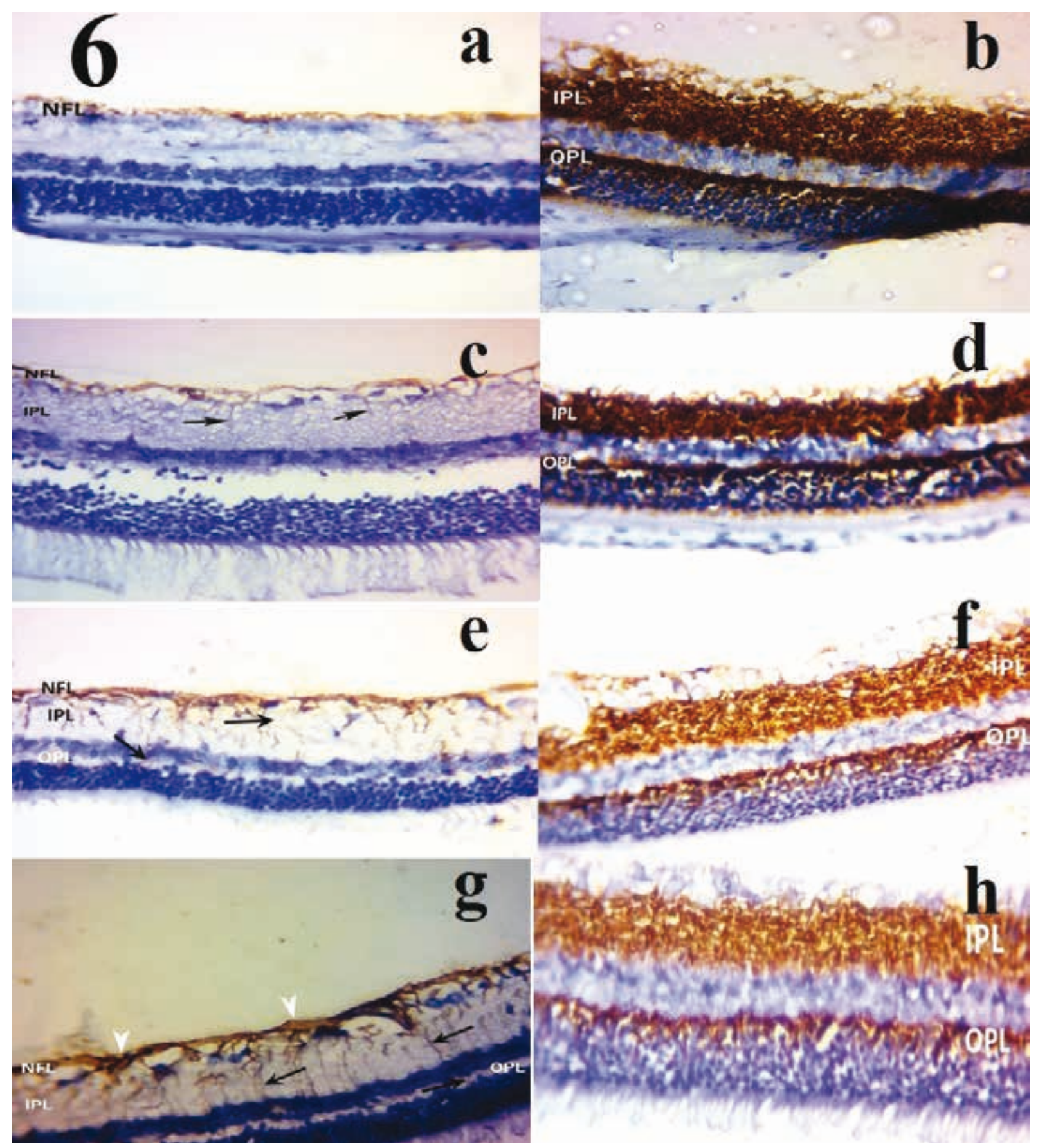

Figure 6. Micrograph of rat retina showing: a. Group I with glial fibrillary acidic protein (GFAP) immunoreactivity is confined to nerve fibre layer (NFL); GFAP $\times 400$ ); b. Group I strong positive brown reaction to synaptophysin (SYN) in both outer (OPL) and inner (IPL) plexiform layers; SYN $\times 400$; c. Group II showing positive immune reaction GFAP in the nerve fibre layer (NFL) and Müller cell processes (arrows) extending into the inner portion of the inner plexiform layer (IPL); GFAP $\times 400$; d. Group II revealing strong immunoreactivity to synaptophysin. in the outer (OPL) and inner (IPL) plexiform layers; SYN $\times 400$; e. Group III showing strong positive reaction at the nerve fibre layer (NFL) and Müller cell processes (arrows) extending through the inner (IPL) and outer plexiform (OPL) layers; GFAP $\times 400$; f. Group III revealing moderate immunoreaction in both inner (IPL) and outer plexiform layer (OPL); SYN $\times 400 ; \mathbf{g}$. Group IV showing numerous Müller cell processes (arrows) extending through the inner (IPL) and outer (OPL) plexiform layers. Also, strong positive reaction of astrocytes (arrowheads) at the nerve fibre layer (NFL) was seen; GFAP $\times 400$; $h$. Group IV showing weak positive immune reaction for synaptophysin, in the inner (IPL) and the outer $(\mathrm{OPL})$ plexiform layers; SYN $\times 400)$. 


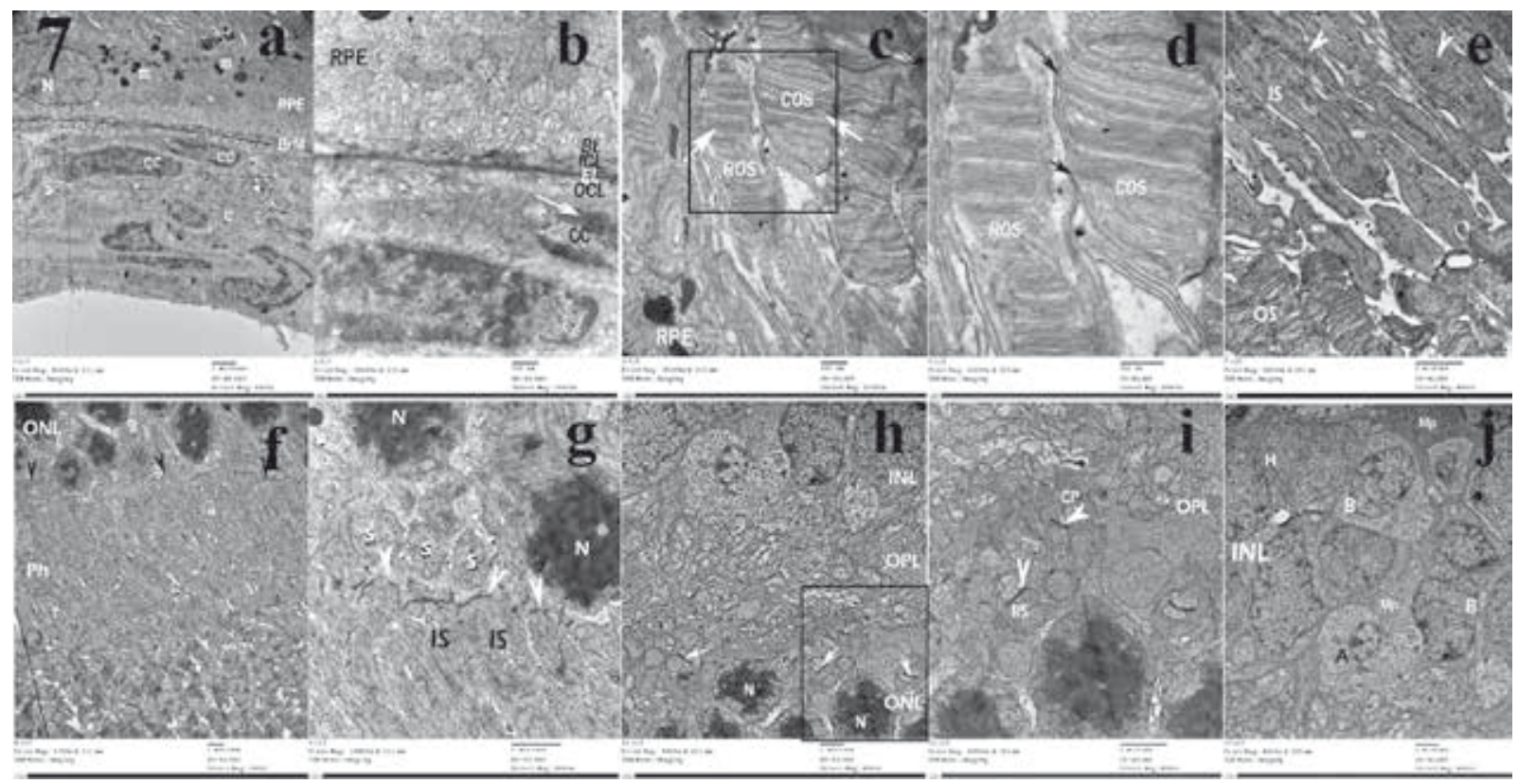

Figure 7. An electron micrograph of rat retina (group I) showing: a. Retinal pigment epithelium (RPE)/Bruch's membrane (BrM)/choriocapillaries (CC) complex. RPE cells have oval euchromatic nucleus $(\mathrm{N})$ and contain melanin granules $(\mathrm{m})$ in their cytoplasm. The BrM separates the pigment epithelium from the choroid and CC; $\mathbf{b}$. The five layers of Bruch's membrane: basal lamina (BL), inner collagenous layer (ICL), elastic lamina (EL), outer collagenous layer (OCL) and basement membrane (arrow) of CC. The retinal pigment epithelium (RPE) lies on the Brush's membrane; c. Photoreceptor rod outer segments (ROS) and cone outer segment (COS) with packed membranous discs (arrows); d. A higher magnification of the inset of Figure 7c revealing the plasmalemmal invaginations (arrows) in the cone outer segment (COS) which are absent in rod outer segment (ROS); e. The photoreceptors outer (OS) and inner (IS) segments. Inner segments show longitudinal arrangement of mitochondria (Mit) and abundant rough endoplasmic reticulum (arrowheads); $f$. The outer nuclear layer (ONL) is separated from the photoreceptor layer $(\mathrm{Ph})$ by the outer limiting membrane (arrowheads). Photoreceptor outer segments (OS) are shown; g. The outer limiting membrane (arrowheads) separating photoreceptor somas (s) and nuclei (N) from photoreceptor inner segments (IS). Photoreceptor nuclei (N) are located at different distance from the outer limiting membrane; $\boldsymbol{h}$. The photoreceptor nuclei (N) forming the outer nuclear layer (ONL). Synaptic ribbons (arrows) are seen within the outer plexiform layer (OPL) which lies between two nuclear layers; the outer (ONL) and the inner (INL) nuclear layers; i. A higher magnification of the inset of Figure 7h revealing the outer plexiform layer (OPL) contains rod spherules (RS) and the cone pedicles (CP). Both contain ribbon synapses (arrowheads); j. different population of cells of inner nuclear layer (INL); bipolar cells (B), amacrine cells $(\mathrm{A})$ and horizontal cell $(\mathrm{H})$. All are surrounded by Müller cell processes (Mp).

\section{Electron microscopic results}

Group I (cortical maturity). The retina of group I showed that the RPE had oval euchromatic nuclei and the cytoplasm contained melanin granules in the apical portion of the cell. Basal infoldings appeared lying on Bruch's membrane which separates the pigment epithelium from the choroid and choriocapillaries (Fig. 7a). Bruch's membrane featured its 5 layers; basal lamina, inner collagenous layer, elastic lamina, outer collagenous layer and basement membrane of choriocapillaries (Fig. 7b).

The photoreceptor outer segments were attached to the RPE. They contained well organised, horizontally aligned membranous discs (Fig. 7c). Outer segments of cones were conical in shape while those of rods were straight (Fig. 7c, d). Cones outer segments featured plasmalemmal invaginations at one side which were absent in rods (Fig. 7d).
The photoreceptors' inner segments contained longitudinal tubular mitochondria and abundant rough endoplasmic reticulum (Fig. 7e). The outer limiting membrane appeared between the outer nuclear layer and the photoreceptor layer (Fig. 7f) separating photoreceptors somas and nuclei from their inner segments (Fig. 7g). Nuclei of rods and cones were located at different distances from the outer limiting membrane (Fig. $7 \mathrm{~g}$ ) forming the outer nuclear layer (Fig. 7h).

The outer plexiform layer revealed the synaptic terminals of photoreceptors with the globular rod terminals (rod spherules) and the expanded cone terminals (cone pedicles) (Fig. 7i). Synaptic terminals contained sharp synaptic ribbons (Fig. 7h, i).

The inner nuclear layer contained heterogeneous population of cells; bipolar cells with heterochromatic nuclei, horizontal cells with euchromatic nuclei and 


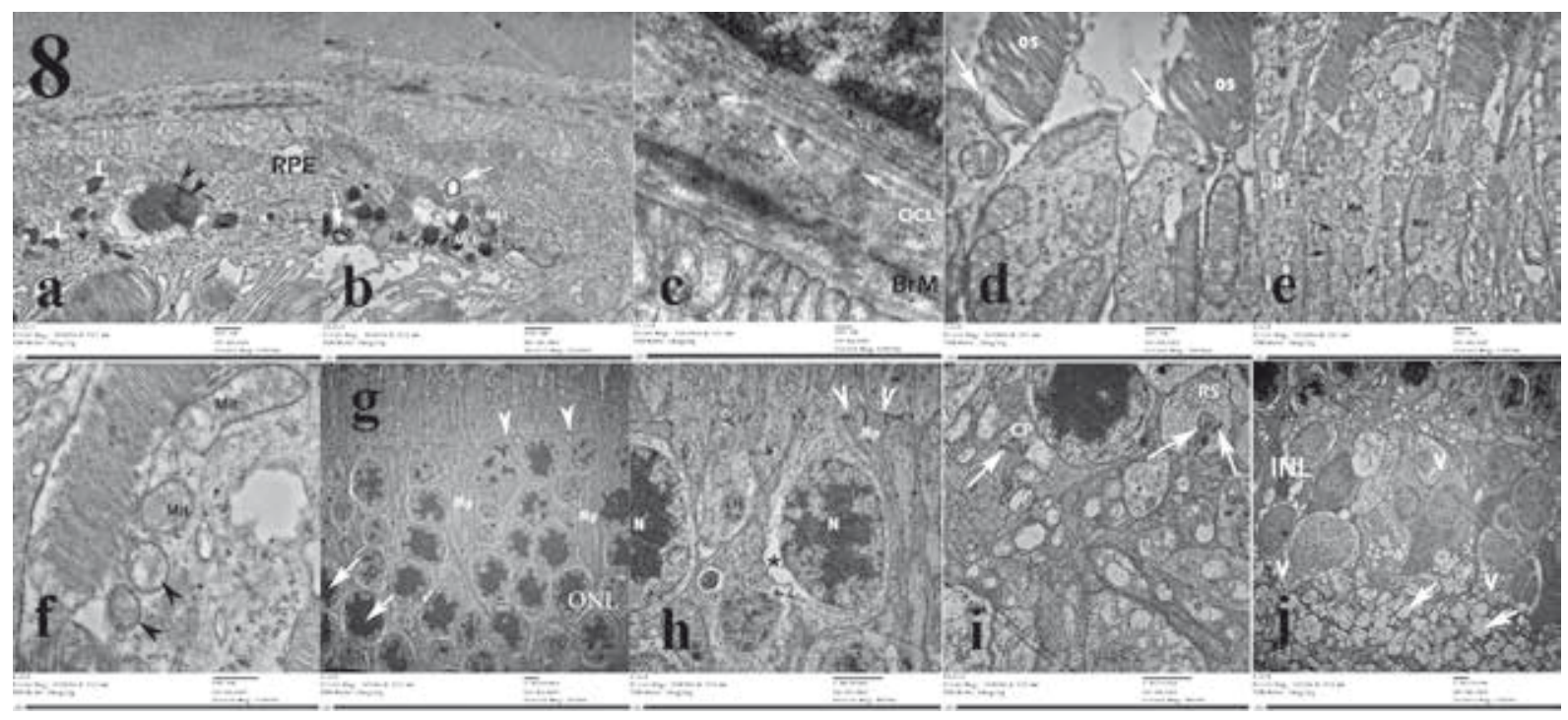

Figure 8. An electron micrograph of rat retina (group II) showing: a. Detached part of membranous discs (arrowheads) within the retinal pigment epithelium (RPE). Lipofuscin granules (L) are seen in the RPE; b. Phagosomes (arrows) and melanolysosomes (MLL) inside a retinal pigment epithelial cell; BrM - Bruch's membrane; c. Electron-dense deposits (arrows) are present in the outer collagenous layer (OCL) of Bruch's membrane (BrM); d. Mild malalignment of membranous discs (arrows) of photoreceptors outer segment (OS); e. The photoreceptor inner segments (IS), containing longitudinally arranged mitochondria (Mit) and well-organized microtubules (arrows); f. Loss of mitochondrial cristae (arrowheads) within the inner segments (IS). Mit — mitochondria; g. Degeneration of few photoreceptor nuclei (arrow) within the outer nuclear layer (ONL); in addition to thickening of Müller cell processes (Mp). Outer limiting membrane (arrowheads) is shown; $\mathbf{h}$. A higher magnification of the Figure $8 \mathrm{~g}$ revealing the photoreceptor nuclei $(\mathrm{N})$ with widening of the perinuclear space $\left({ }^{*}\right)$. Müller cell processes (MP) intervening the space between photoreceptor somas, with their apical processes form the outer limiting membrane (arrowheads); i. The rod spherules (RS) and the cone pedicles (CP). Synaptic ribbons (arrows) appear as sharp well-defined bars; j. Vacuolations (V) in the cells of inner nuclear layer (INL) with decreased electro-density of their axons (arrows) in the inner plexiform layer.

amacrine cells with heterochromatic nuclei and pale indented cytoplasm. The Müller cell processes were filling the background between other cells (Fig. 7j).

Group II (middle-aged). Examination of the different layers of the retinae of group II revealed mild changes. Some of the RPE cells contained detached parts of outer segment membranous discs and lipofuscin granules (Fig. 8a). Phagosomes appeared within the cells, in addition to melanolysosomes (Fig. 8b).

Bruch's membrane showed electron-dense deposits, especially in outer collagenous layer (Fig. 8c). The membranous discs of photoreceptor outer segments showed mild malalignment in some specimens (Fig. 8d); the inner segments showed apparently normal arrangement of microtubules and longitudinally settled mitochondria (Fig. 8e) with occasional loss of mitochondrial cristae (Fig. 8f). The outer nuclear layer displayed degeneration of few photoreceptor nuclei, concomitant with thickening of Müller cell processes which form the matrix background between the photoreceptor nuclei (Fig. 8g). Widening of the perinuclear space of photoreceptor nuclei was occasionally noticed (Fig. 8h). The synaptic ribbons appeared sharp and well defined inside the photoreceptors synaptic terminals in both rod spherules and cone pedicles (Fig. 8i). Some cells of the inner nuclear layer exhibited vacuolations (Fig. 8j) with decrease in the electron density of their axons located in the inner plexiform layer (Fig. 8j).

Group III (aged). The RPE presented whirling of basal folds, numerous phagosomes (Fig. 9a) as well as lipofuscin and melanolipofuscin granules (Fig. 9b). Filling of the subretinal space was encountered (Fig. 9b) together with poor attachment of outer segments to the RPE (Fig. 9b). Thickening of Bruch's membrane was encountered, in addition to dilatation of choriocapillaries (Fig. 9c). Choriocapillaries wall presented proliferation of the basal lamina, degeneration of the endothelial cells and extrusion of pericytes were observed in (Fig. 9d). The photorecerptor outer segment showed malalignment of the membranous discs, in addition to areas of distorted discs (Fig. 9e). The photoreceptor inner segments contained long convoluted mitochondria (Fig. 9f). Defective mitochondrial cristae, numerous phagosomes 


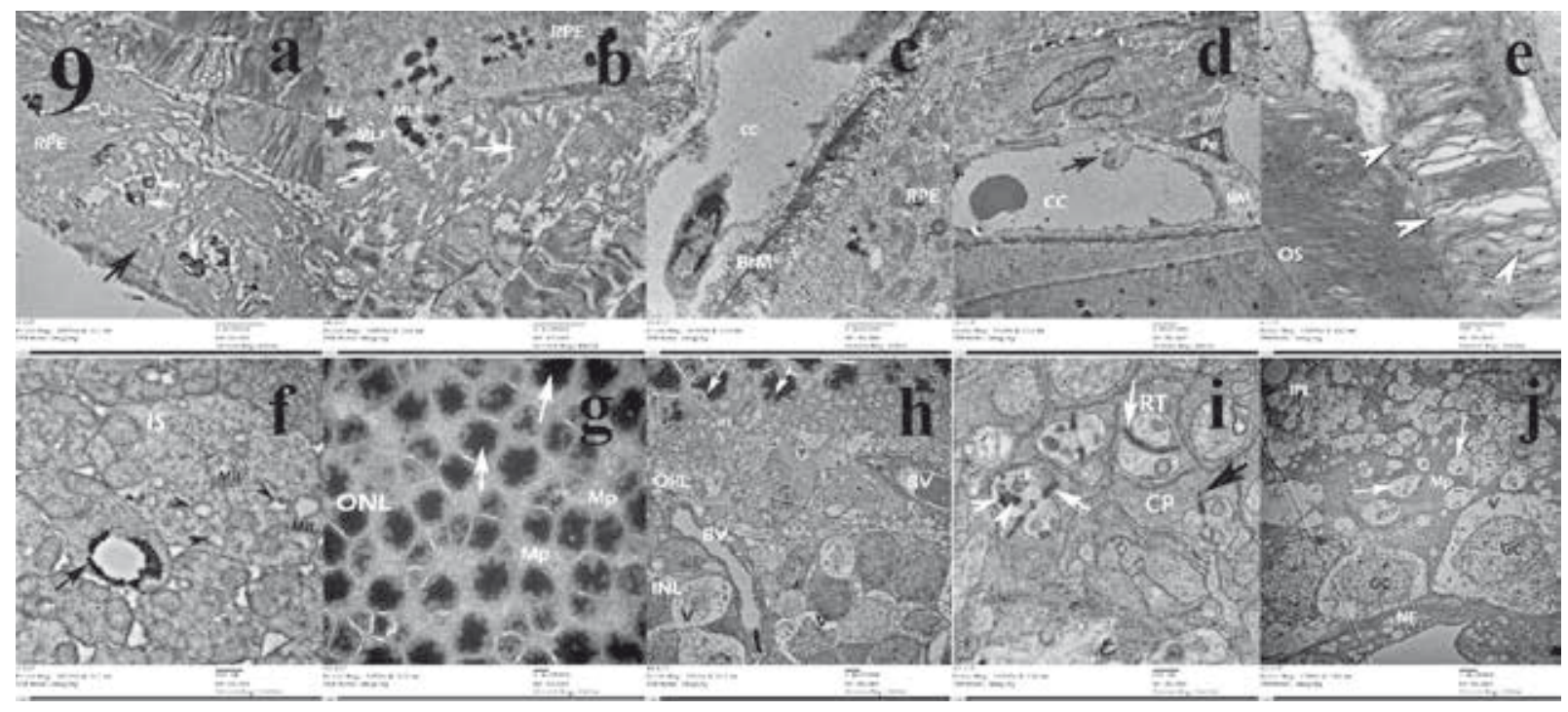

Figure 9. A photomicrograph of rat retina (group III) showing: a. Whirling of basal folds (black arrow), numerous phagolysosomes (white arrows) in the retinal pigment epithelium (RPE); $\boldsymbol{b}$. That the subretinal space is filled (arrows). Retinal pigment epithelium (RPE) exhibits numerous lipofuscin (LF) and melanolipofuscin (MLF) granules; c. Thickening of the Bruch's membrane (BrM) and dilated choriocapillaries (CC). Retinal pigment epithelium (RPE) is seen; $\boldsymbol{d}$. Dilatation of choriocapillaries (CC) with proliferation of the basement membrane (BM) and extrusion of pericytes (Pe). Projection inside the vessel lumen was observed (arrow); e. Distortion of the membranous discs (arrowheads); f. Numerous phagosomes (arrowheads), defective mitochondrial cisterns (Mit) and dilated congested blood vessel (arrow) are seen in inner segments (IS); g. Cells of the outer nuclear layer (ONL) are variable-sized with the intervening Müller cell processes (Mp) appear thicker. The photoreceptor nuclei show degeneration (arrows); $\mathbf{h}$. Degeneration of photoreceptor nuclei (arrows), associated with low electron-density of rod terminals (RT). Dilated blood vessels (BV) are seen in outer plexiform layer (OPL) encroaching the inner nuclear layer (INL). Cells of inner nuclear layer show sever vacuolation (V); i. Swollen synaptic ribbons (arrows) in the rod terminals (RT) and fragmented ribbons (black arrow) in the cone pedicle (CP) were encountered. Lipofuscin (LF) granules and inclusion bodies (arrowheads) were seen in the rod terminals; j. Ganglion cells (GC) with cytoplasmic vacuolation (V). Müller cell processes (Mp) are highly electron-dense together with faint axons (arrows) in the inner plexiform layer (IPL). Nerve fibre layer (NF) is seen.

and dilated congested blood vessels were seen within inner segments (Fig. 9f).

Cells of the outer nuclear layer were of variable size separated with broadened Müller cell processes (Fig. 9g). Some nuclei displayed degeneration (Fig. $9 g, h)$. The outer plexiform layer showed decreased electron-density of the rod terminals (Fig. 9h). Synaptic ribbons decreased in number and appeared fragmented (Fig. 9i) and swollen (Fig. 9i). Rod synaptic terminals contained lipofuscin granules and inclusion bodies (Fig. 9i). Dilated blood vessels were seen in the outer plexiform layer encroaching the inner nuclear layer. Their basement membranes displayed thickening and lamination (Fig. 9h). Inner nuclear layer cells exhibited severe vacuolations (Fig. 9h). Müller cell processes were highly electron-dense between faint axons in the inner plexiform layer (Fig. 9j). Ganglion cells presented severe cytoplasmic vacuolation (Fig. 9j).

Group IV (senile). Retinal pigment epithelium showed numerous large lipofuscin and melanoli- pofuscin granules (Fig. 10a). The subretinal space appeared obliterated with dense microvilli sent from the apical aspect of the RPE (Fig. 10b). The photoreceptor outer segments showed major disorganisation of the membranous discs (Fig. 10c) Moreover, empty portions within the plasmalemmal envelops were seen (Fig. 10a, c).

The outer limiting membrane was thickened as the apical portions of Müller cell processes were thickened (Fig. 10d); also breaks in the OLM were encountered (Fig. 10e). The photoreceptor nuclei exhibited extensive degeneration (Fig. 10e, $f, g$ ) with widening of the perinuclear space (Fig. 10f, g). The outer plexiform layer appeared as a thin layer of low electron-dense synaptic terminals (Fig. 10g). The synaptic terminals revealed evident loss of synaptic vesicles and fragmentation of the synaptic ribbons (Fig. 10h). Inner nuclear layer cells exhibited severe vacuolations with poor cytoplasmic outlines in some cells (Fig. 10i, j). The synaptic terminals of the bipolar and amacrine cells appeared as empty figures (Fig. 10j). Decreased 

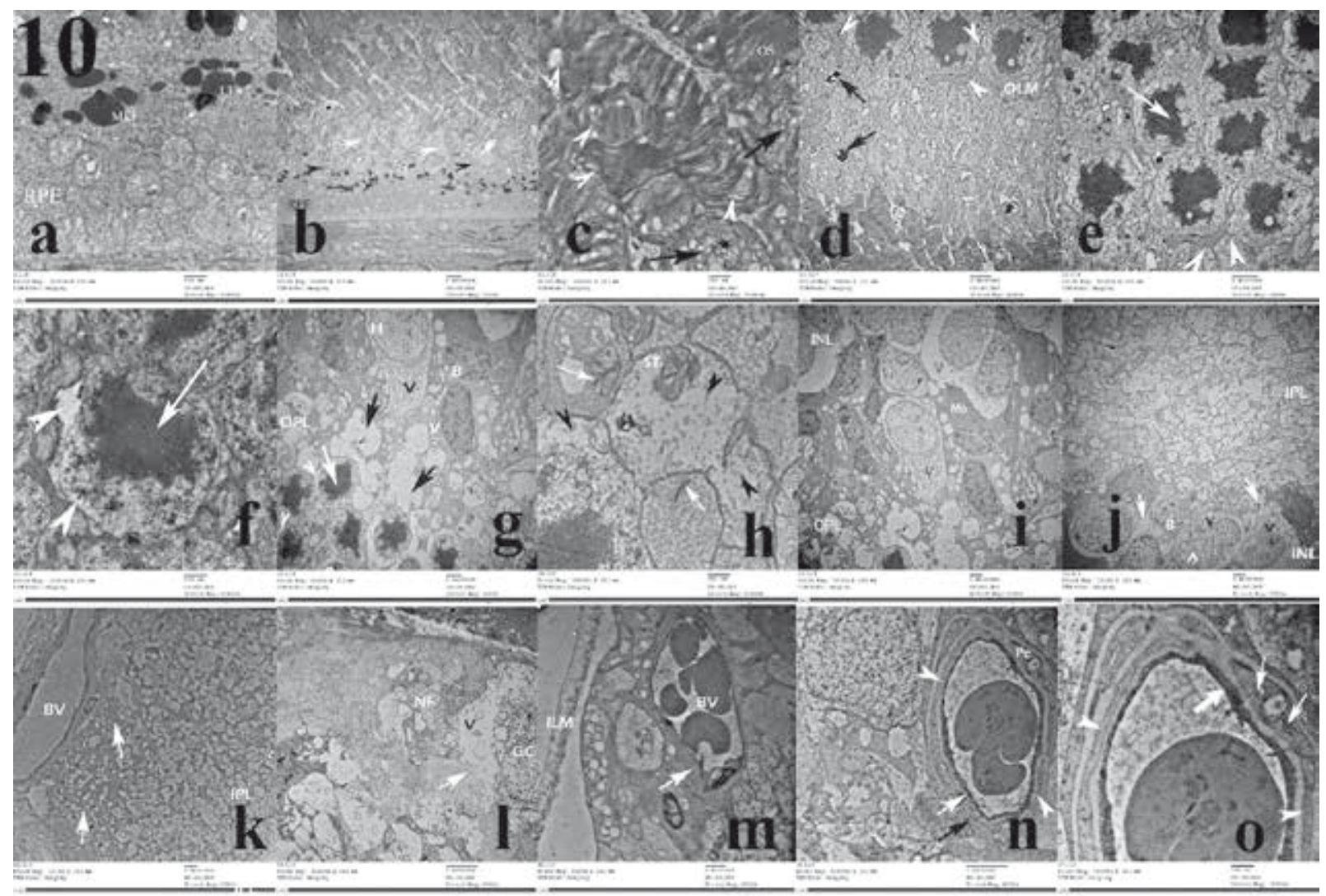

Figure 10. A photomicrograph of rat retina (group IV) showing: a. Numerous lipofuscin (LF) and melanolipofuscin (MLF) granules are seen in the retinal pigment epithelium (RPE); $\mathbf{b}$. The photoreceptors/ retinal pigment epithelium (RPE)/Bruch's membrane complex with empty plasmalemmal chambers (arrows) and obliterated subretinal space (arrowheads). The photoreceptors outer segments (OS) are seen; c. Empty potions (arrows) within the photoreceptor outer segments (OS) together with sever distortion of (arrowheads) the remaining discs; $\mathbf{d}$. Thick, electro-dense Müller cell processes (arrowheads) causing thickening of the outer limiting membrane. Congested blood vessels (arrows) are seen; e. A higher magnification of the previous figure. The photoreceptor nuclei exhibit degeneration (arrow). Breaks in the outer limiting membrane (arrowheads) are encountered; $f$. Distorted nuclear envelop and increased perinuclear space (arrowheads) in addition to nuclear degeneration (arrow); g. Ghost-like synaptic terminals are seen in the outer plexiform layer (black arrows), vacuolations (V) in bipolar (B) and horizontal $(\mathrm{H})$ cells, degeneration of photoreceptor nuclei (white arrow) and increased perinuclear space (arrowheads); $\mathbf{h}$. Marked loss of synaptic vesicles (arrowheads) and fragmentation of the synaptic ribbons (arrows) inside the synaptic terminals (ST); i. That the cells of the inner nuclear layer (INL) exhibit cytoplasmic vacuolations (V). Müller cell processes (MP) are of high electron density. Synaptic terminals are seen within the outer plexiform layer (OPL); j. The inner nuclear layer (INL) with bipolar (B) and amacrine (A) cells show severe vacuolation (V) with poor cytoplasmic outlines of the cells (arrows). The synaptic terminals of both cells appear as empty-like figures in the inner plexiform layer (IPL); k. A dilated blood vessels (BV) in the ganglion cell layer. Together with the empty figures of bipolar axons and areas of decreased arborisation (arrows) in the inner plexiform layer (IPL); I. Disrupted cytoplasmic membrane (arrow) of retinal ganglion cell (GC) together with sever vacuolation (V). Nerve fibre (NF) bundles are apparently smaller; $\mathbf{m}$. A dilated congested blood vessel (BV) with thick basement membrane (arrow). Thick detached inner limiting membrane (ILM) is seen; $\mathbf{n}$. Thickening of the internal (white arrow) and external (black arrow) basement membranes. Pericytes (Pe) send cytoplasmic processes (arrowheads) within the thickened basement membrane. Endothelial cells are degenerated and can't be seen; $\mathbf{0}$. The previous figure at a higher magnification. Pericytes show electro-dense inclusions (arrows) and long cytoplasmic processes (arrowheads) within the vessel basement membrane. Marked proliferation of capillary basal lamina is demonstrated (thick arrow).

arborisation (Fig. 10k) were encountered in the inner plexiform layer. Disrupted cytoplasmic membrane of retinal ganglion cells together with severe vacuolation were characteristic. Nerve fibre bundles were apparently smaller (Fig. 10l). Thickening and detachment were encountered in other areas (Fig. 10m).

The ganglion cell layer showed dilatation and congestion of blood vessels (Fig. $10 \mathrm{~m}$ ). In the blood vessels of different retinal layers, both internal and external basement membranes were thickened, along with endothelial cells degeneration (Fig. 10n) and basal lamina proliferation. Pericytes sent cytoplasmic processes extending through the thickened basement membrane (Fig. 10n, o) and exhibited electron-dense cytoplasmic inclusions (Fig. 10o). 
Table 1. The mean thickness of different layers of the retina in different age groups

\begin{tabular}{lcccc}
\hline Parameter & Group I & Group II & Group III & Group IV \\
\hline ILM-OLM thickness $[\mu \mathrm{m}]$ & $152.25 \pm 17.30$ & $159.21 \pm 21.62$ & $112.99 \pm 5.90$ & $102.08 \pm 23.44$ \\
IPL thickness $[\mu \mathrm{m}]$ & $73.92 \pm 6.29$ & $76.41 \pm 9.27$ & $49.51 \pm 3.27$ & $43.95 \pm 4.56$ \\
INL thickness $[\mu \mathrm{m}]$ & $30.04 \pm 3.03$ & $29.63 \pm 6.26$ & $18.09 \pm 2.36$ & $11.78 \pm 4.05$ \\
OPL thickness $[\mu \mathrm{m}]$ & $12.44 \pm 1.75$ & $11.88 \pm 3.19$ & $9.43 \pm 1.61$ & $6.26 \pm 1.65$ \\
ONL thickness $[\mu \mathrm{m}]$ & $47.07 \pm 2.08$ & $38.11 \pm 6.65$ & $33.01 \pm 4.94$ & $33.98 \pm 9.39$ \\
\hline
\end{tabular}

Data are shown as mean \pm standard deviation. ILM-OLM — inner limiting membrane-outer limiting membrane; IPL — inner plexiform layer; INL — inner nuclear layer; OPL — outer plexiform layer; ONL — outer nuclear layer

Table 2. Comparison of the mean thickness of the inner limiting membrane-outer limiting membrane (ILM-OLM) among the different age groups

\begin{tabular}{lccc}
\hline Group & ILM-OLM thickness $[\mu \mathrm{m}]$ (mean \pm SD) & Comparative groups & P (ANOVA test) \\
\hline Group I & $152.25 \pm 17.30$ & Group II & 1.000 \\
& & Group III & $0.000^{*}$ \\
& & Group IV & $0.000^{*}$ \\
Group II & $159.21 \pm 21.62$ & Group I & 1.000 \\
& & Group III & $0.000^{*}$ \\
& & Group IV & $0.000^{*}$ \\
Group III & $112.99 \pm 5.90$ & Group I & $0.000^{*}$ \\
& & Group II & $0.000^{*}$ \\
Group IV & & Group IV & 0.230 \\
& & Group I & $0.000^{*}$ \\
\hline
\end{tabular}

${ }^{*}$ p-value $\leq 0.05$ statistically significant; SD - standard deviation

\section{Statistical and histomorphometric results}

ILM-OLM thickness. The mean thickness of ILM-OLM in group I was $152.25 \pm 17.30 \mu \mathrm{m}$ and in group II was $159.21 \pm 21.62 \mu \mathrm{m}$. The difference in the mean thickness between the two groups was found to be statistically non-significant ( $p=1.0$ ) (Tables 1, 2; Fig. 11A). The mean thickness of ILM-OLM in group III was $112.99 \pm 5.90 \mu \mathrm{m}$. The mean thickness was less in group III as compared to group I and group II and these difference were found to be statistically significant $(p=0.000)$. In group IV, the mean thickness of ILM-OLM was $102.08 \pm 23.44 \mu \mathrm{m}$. There was age related statistical significant decrease in the thickness of ILM-OLM as compared to group I and group II $(p=0.000)$ for both. The difference in the mean thickness of ILM-OLM between group III and group IV was found to be statistically nonsignificant $(p=0.2)$.
IPL thickness. The mean thickness of IPL was $73.92 \pm 6.29 \mu \mathrm{m}$ and it was non-significantly different from IPL thickness which was $76.41 \pm$ \pm 9.27 (Tables 1, 3; Fig. 11B). The mean thickness of the IPL significantly decreased in group III to be $49.51 \pm$ \pm 3.27 ; as compared with group I and II $(p=0.000$ and $p=0.000$ ). IPL mean thickness became $43.95 \pm$ \pm 4.56 in group IV. This found to be highly statistically significant as compared with groups I, II and III ( $p=0.000, p=0.000$ and $p=0.014$, respectively).

INL thickness. Group I mean INL thickness was $30.04 \pm 3.03$ and it was $29.63 \pm 6.26$ in group II $(p=1.0)$. INL of group III decreased significantly as compared with group I and II ( $p=0.000)$ for both (Tables 1, 4; Fig. 11C). INL thickness in group IV was $11.78 \pm 4.05$. There was age related significant difference between group IV and group I ( $p=0.000)$ and significant difference between group IV and group III ( $p=0.000)$. 

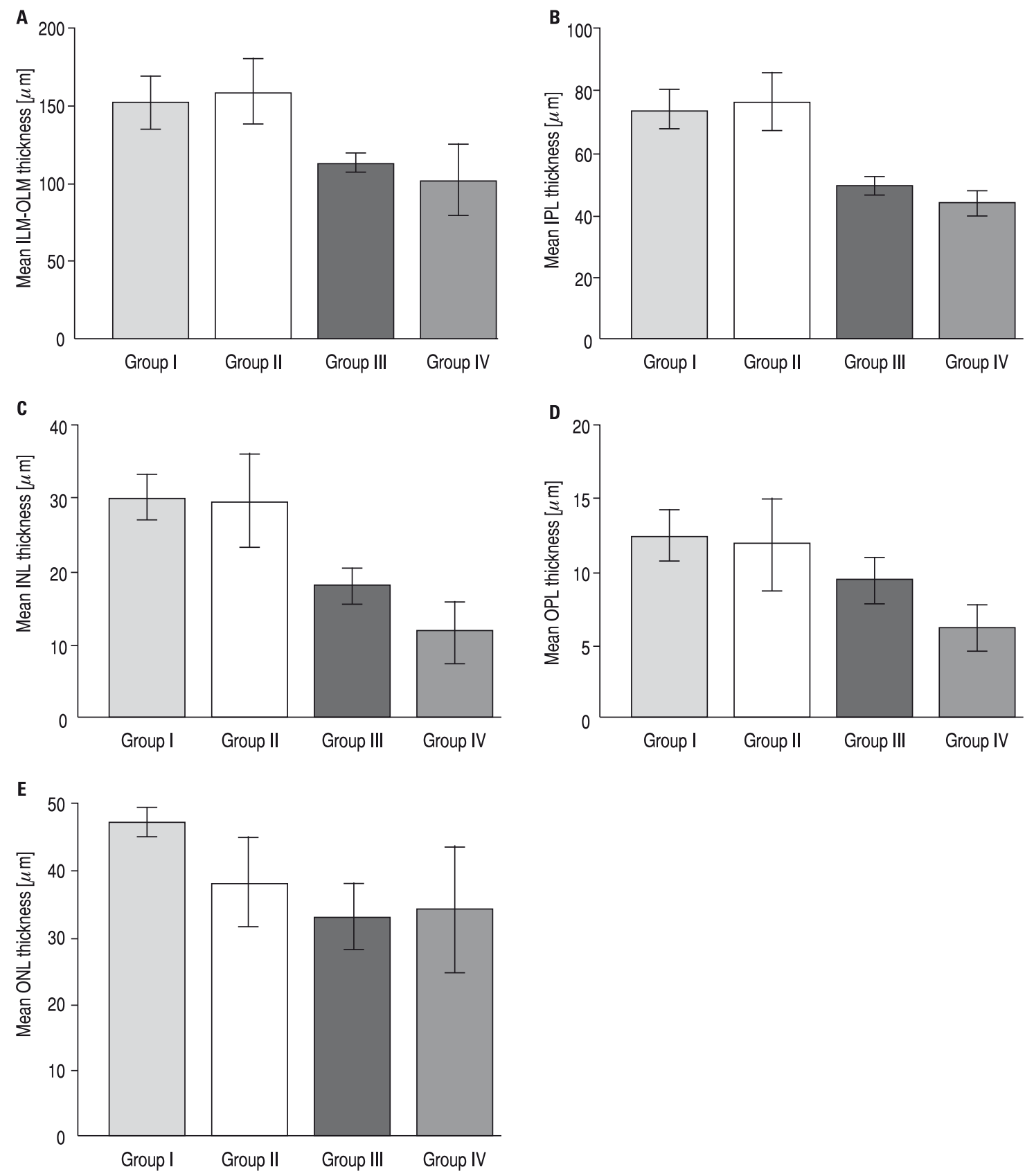

Figure 11. The mean thickness in the different groups (error bars: \pm standard deviation); A. Inner limiting membrane-outer limiting membrane (ILM-OLM); B. Inner plexiform layer (IPL); C. Inner nuclear layer (INL); D. Outer plexiform layer (OPL); E. Outer nuclear layer (ONL).

OPL thickness. The mean thickness was $12.44 \pm$ \pm 1.75 in group I, $11.88 \pm 3.19$ in group II, $9.43 \pm$ \pm 1.61 in group III and $6.26 \pm 1.65$ in group IV. The difference between group I and II was statistically non-significant; while a statistically significant decrease was calculated between group II and III $(p=0.001)$ and between III and IV $(p=0.000)$ (Table 5; Fig. 11D).
ONL thickness. The mean thickness of ONL was $47.07 \pm 2.08$ in group I and was $38.11 \pm 6.65$ in group II. The mean thickness decreased to $33.01 \pm$ \pm 4.94 in group III; this was statistically significant when compared with group I and II ( $p=0.000$ and 0.033 respectively) (Table 6, Fig. 11E). There was a highly significant decrease in ONL thickness between group IV and group I ( $p=0.000)$, while the 
Table 3. Comparison of the mean thickness of the inner plexiform layer (IPL) among the different age groups

\begin{tabular}{lccc}
\hline Group & IPL thickness $[\boldsymbol{\mu m}]$ (mean \pm SD) & Comparative groups & P (ANOVA test) \\
\hline Group I & $73.92 \pm 6.29$ & Group II & 0.972 \\
& & Group III & $0.000^{*}$ \\
& & Group IV & $0.000^{*}$ \\
Group II & $76.41 \pm 9.27$ & Group I & 0.972 \\
& & Group III & $0.000^{*}$ \\
& & Group IV & $0.000^{*}$ \\
Group III & $49.51 \pm 3.27$ & Group I & $0.000^{*}$ \\
& & Group II & $0.000^{*}$ \\
Group IV & & Group IV & $0.014^{*}$ \\
& $43.95 \pm 4.56$ & Group I & $0.000^{*}$ \\
\hline
\end{tabular}

${ }^{*} \mathrm{p}$-value $\leq 0.05$ statistically significant; SD - standard deviation

Table 4. Comparison of the mean thickness of the inner nuclear layer (INL) among the different age groups

\begin{tabular}{lccc}
\hline Group & INL thickness $[\mu \mathrm{m}]$ (mean \pm SD) & Comparative groups & P (ANOVA test) \\
\hline Group I & $30.04 \pm 3.03$ & Group II & 1.000 \\
& & Group III & $0.000^{*}$ \\
& & Group IV & $0.000^{*}$ \\
Group II & $29.63 \pm 6.26$ & Group I & 1.000 \\
& & Group III & $0.000^{*}$ \\
& & Group IV & $0.000^{*}$ \\
Group III & $18.09 \pm 2.36$ & Group I & $0.000^{*}$ \\
& & Group II & $0.000^{*}$ \\
Group IV & & Group IV & $0.000^{*}$ \\
& $11.78 \pm 4.05$ & Group I & $0.000^{*}$ \\
\hline
\end{tabular}

${ }^{*} \mathrm{p}$-value $\leq 0.05$ statistically significant; $S D-$ standard deviation

Table 5. Comparison of the mean thickness of the outer plexiform layer (OPL) among the different age groups

\begin{tabular}{lccc}
\hline Group & OPL thickness $[\mu \mathrm{m}]$ (mean \pm SD) & Comparative groups & P (ANOVA test) \\
\hline Group I & $12.44 \pm 1.75$ & Group II & 1.000 \\
& & Group II & $0.000^{*}$ \\
& & Group IV & $0.000^{*}$ \\
Group II & $11.88 \pm 3.19$ & Group I & 1.000 \\
& & Group III & $0.001^{*}$ \\
& & Group IV & $0.000^{*}$ \\
Group III & $9.43 \pm 1.61$ & Group I & $0.001^{*}$ \\
& & Group II & $0.000^{*}$ \\
Group IV & & Group IV & $0.000^{*}$ \\
& $6.26 \pm 1.65$ & Group I & $0.000^{*}$ \\
\hline
\end{tabular}


Table 6. Comparison of the mean thickness of the outer nuclear layer (ONL) among the different age groups

\begin{tabular}{lccc}
\hline Group & ONL thickness $[\mu \mathrm{m}]($ mean \pm SD) & Comparative groups & P (ANOVA test) \\
\hline Group I & $47.07 \pm 2.08$ & Group II & $0.000^{*}$ \\
& & Group II & $0.000^{*}$ \\
& & Group IV & $0.000^{*}$ \\
Group II & $38.11 \pm 6.65$ & Group I & $0.000^{*}$ \\
& & Group III & $0.033^{*}$ \\
& & Group IV & 0.140 \\
Group III & $33.01 \pm 4.94$ & Group I & $0.000^{*}$ \\
& & Group II & $0.033^{*}$ \\
Group IV & & Group IV & 1.000 \\
& $33.98 \pm 9.39$ & Group I & $0.000^{*}$ \\
\hline
\end{tabular}

${ }^{*} \mathrm{p}$-value statistically significant; SD — standard deviation

Table 7. Comparison of the mean optical density of synaptophysin among the different age groups

\begin{tabular}{lccc}
\hline Group & Synaptophysin optical density (mean \pm SD) & Comparative groups & P (ANOVA test) \\
\hline Group I & $0.94 \pm 0.03$ & Group II & $0.000^{*}$ \\
& & Group III & $0.000^{*}$ \\
& & Group IV & $0.000^{*}$ \\
Group II & $0.79 \pm 0.03$ & Group I & $0.000^{*}$ \\
& & Group III & $0.000^{*}$ \\
& & Group IV & $0.000^{*}$ \\
Group III & $0.66 \pm 0.03$ & Group I & $0.000^{*}$ \\
& & Group II & $0.000^{*}$ \\
Group IV & & Group IV & $0.000^{*}$ \\
& & Group I & $0.000^{*}$ \\
\hline
\end{tabular}

${ }^{*} \mathrm{p} \leq 0.05$ statistically significant; $\mathrm{SD}$ - standard deviation
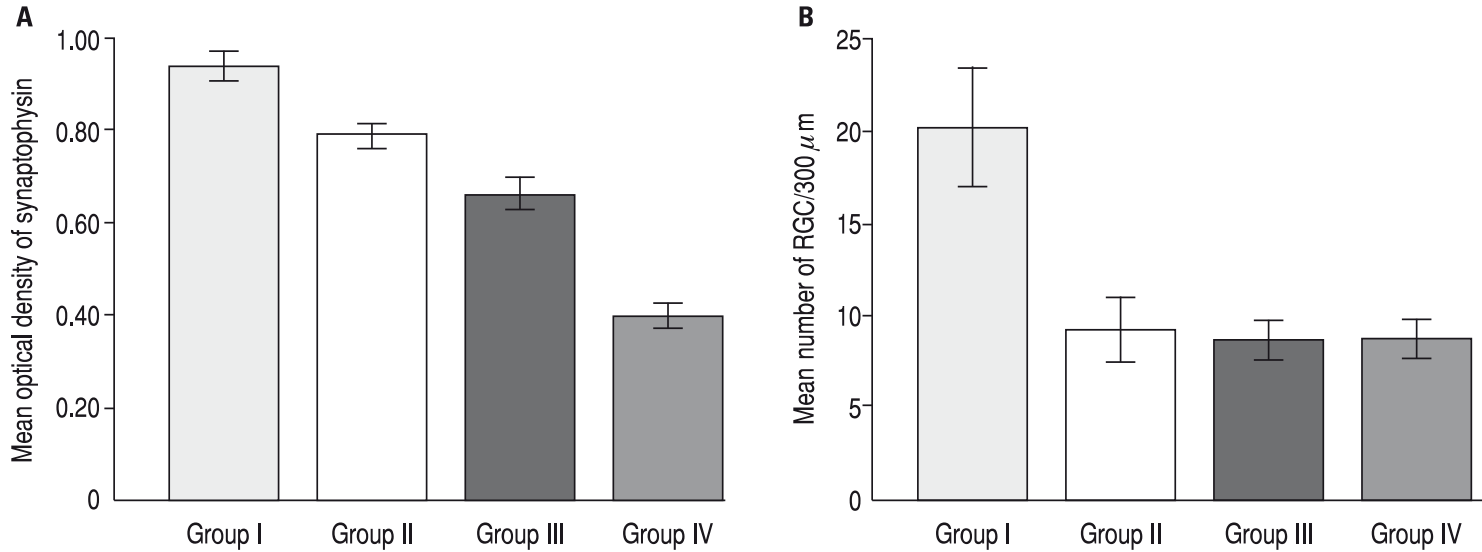

Figure 12. The mean (error bars: \pm standard deviation); A. Optical density of synaptophysin in the different groups; B. Number of the retinal ganglion cells $(\mathrm{RGC}) / 300 \mu \mathrm{m}$ in the different groups. 
Table 8. Comparison of the mean number of retinal ganglion cells (RGC) layer among the different age groups

\begin{tabular}{lccc}
\hline Group & Number of RGC/300 $\mu \mathrm{m}$ (mean \pm SD) & Comparative groups & P (ANOVA test) \\
\hline Group I & $20.20 \pm 3.19$ & Group II & $0.000^{*}$ \\
& & Group III & $0.000^{*}$ \\
& & Group IV & $0.000^{*}$ \\
Group II & $9.20 \pm 1.76$ & Group I & $0.000^{*}$ \\
& & Group III & 1.000 \\
& & Group IV & 1.000 \\
Group III & $8.60 \pm 1.04$ & Group I & $0.000^{*}$ \\
& & Group II & 1.000 \\
& & Group IV & 1.000 \\
Group IV & & Group I & $0.000^{*}$ \\
& & Group II & 1.000 \\
\hline
\end{tabular}

${ }^{*} \mathrm{p} \leq 0.05$ statistically significant; $\mathrm{SD}$ - standard deviation

difference between group IV and group II ( $p=0.14)$ and between group IV and group III ( $p=1.00)$ was found to be statistically non-significant.

Synaptophysin optical density. The mean density of synapses was $0.94 \pm 0.03$ in group l, $0.79 \pm$ \pm 0.03 in group II, $0.66 \pm 0.03$ in group III and $0.40 \pm 0.03$ in group IV. The difference among the different groups was found statistically significant (Table 7, Fig. 12A).

Ganglion cell count. The mean number of retinal ganglion cells $/ 300 \mu \mathrm{m}$ in group I was $20.20 \pm 3.19$ (Table 8, Fig. 12B). Group II presented a statistically significant decrease in ganglion cell count to be $9.20 \pm 1.76(p=0.000)$ (Table 8, Fig. 12B). The mean number of retinal ganglion cells in group III was $8.60 \pm 1.04$ and in group IV was $8.60 \pm 1.04$. The difference between groups II, III and IV were found to be statistically non-significant.

\section{DISCUSSION}

In this work, effect of age on the morphology of retinal layers was evident at the light, electron microscopic and immunohistochemical levels. The changes were encountered both in RPE and retinal neurons. Retinal neurons exhibited numerous agerelated quantitative and qualitative alterations in cells and synapses, some of which could underlie declines in visual acuity.

In the present study, although light histological findings were scarce, the ultrastructural examination revealed prominent changes in the retinal pigment epithelial layer during early phases of aging. Signs included presence of numerous phagolysosomes and melanolysosomes in addition to exaggerated basal infoldings. In the aged rat retina, whirling extensions of the basal membrane into the cytoplasm were characteristic in the RPE cells. These findings were consistent with the data provided by Boya et al. [6] who observed progressive changes in the RPE/ Bruch's complex in aged Fischer rats. In the present work, changes in pigmentation were among the most significant and noticeable changes to occur in RPE cells with age. During normal aging, the appearance of the RPE layer was altered; there was a decrease in the number of melanosomes and an increase in the number of lipofuscin granules. These results were in agreement with results of Boya et al. [6] who reported accumulation of lipids in the RPE, for which the autofluorescence was indicative of lipofuscin. Boulton [5] submitted that RPE lipofuscin is a result of both the incomplete degradation of phagocytosed photoreceptor outer segments and autophagic removal of damaged organelles-protein aggregates. Keeling et al. [20] added that chronic degeneration of the RPE is a precursor to pathological changes in the outer retina. Impaired handling and processing in the endocytic/phagosome and autophagy pathways lead to the accumulation of lipofuscin and chemically modified compounds within RPE. These contribute to increased proteolytic and oxidative stress, resulting in irreversible damage to post-mitotic RPE cells. 
Heterogeneous deposits in the form of melanolipofucin granules were found in the present work to increase within the RPE with age. Lipofuscin and melanolipofuscin granules started to appear not only in the aged group, but also in the middle-aged group. This was consistent with a manuscript published by Warrburton et al. [35] which detected the accumulation of melanolipofuscin in human RPE from different decades of life and assessed their phototoxicity to RPE cultures in vitro.

In the present study, senile group sections revealed rarity of melanin granules and the remaining melanin granules were conjugated with lipofuscin granules. Bonilha [4] suggested that melanolipofuscin might not originate from photoreceptor outer segments phagocytosis but melanolipofuscin accumulates as a result of the melanosomal autophagocytosis of RPE cells. That hypothesis was supported by the absence of photoreceptor-specific proteins on analysis of the composition of melanolipofuscin. Studies have suggested that various components of these heterogeneous lipofuscin deposits may drive immune dysregulation via monocyte and microglial activation [24].

The present work revealed thickening of the Bruch's membrane that was obvious and progressive with aging. Electro-dense deposits were noticed especially in the inner collagenous layer, starting from the 12 months group. This was also reported by Szabadfi et al. [34] who clarified that aged Bruch's membrane displays an increase in phospholipids, triglycerides, fatty acids and free cholesterol content which called advanced glycation end products.

In this work, dilated choriocapillaries with proliferation of the basal lamina, degeneration of endothelial cells and extrusion of pericytes were observed. Fibrosis of the choriocapillaries was profound especially in the senile group. Chirco et al. [10] elucidated that the choriocapillaries are especially susceptible to hypoxia. This dense layer allows the passage of oxygen and nutrients to the RPE and photoreceptor cells and also removes waste products via systemic circulation. They added that loss of the lining endothelial cells is a key contributor to the development of age-related retinal degenerative changes.

The present study found that the photoreceptors were evidently affected as the age increased. The photoreceptor outer segments showed attenuated striations on the light microscopic level. On the electron microscopic level, the middle-aged group featured loss of the proper alignment of photoreceptors mem- branous discs in few outer segments that progressed to presence membranous whorls in the aged group. The affection in the senile group was profound so that areas devoid of the discs inside the plasmalemmal envelops were encountered. Disorganised discs were also reported by Nag and Wadhwa [28], who studied the structural alterations of aging human retina and linked these changes to the effect of oxidative stress, inflammation and chronic light exposure. Furthermore, in the present work, photoreceptor inner segments presented numerous phagosomes, long convoluted mitochondria and defective mitochondrial cisterns. Similar changes were reported by Litts et al. [22]. Nag and Wadhwa [28] suggested that the occurrence of aging changes of mitochondria might be due to increased mitochondrial vulnerability via oxidative stress, light or toxic substances. Such changes might cause energy depletion and photoreceptor loss in human retina with aging.

Transmission electron microscopy in the present work revealed thickening of the OLM. In the senile group, there was prominent thickening of the apical part of Müller cell processes, which form a network of adherent junction complexes that form the OLM. In addition, breaks and interruption of the OLM were encountered. Hippert et al. [17] gave enlightenment that photoreceptor death is likely to disrupt the OLM and compromises the orientation and polarity of the photoreceptors.

In the present work, the retinal (OLM-ILM) thickness displayed an initial increase in group II which proved to be statistically non-significant. Then, the retinal thickness of group III decreased significantly by $25.7 \%$ and further thinned by $32.9 \%$ of its original thickness in group I. Significant thinning of the retina was also observed by Nadal-Nicolás et al. [27]. On the contrary, Szabadfi et al. [34] found increased OLM-ILM distance and IPL thickness in 36-month-old degu rat retinae. The authors explained their findings by the loose retinal structure observed in aging retinae.

In the present work, the outer nuclear layer showed spacing between the photoreceptor nuclei. Some nuclei appeared fragmented and pyknotic on light microscopic examination and degenerated on electron microscopic examination with widening of the perinuclear space. These changes appeared limited in group II and became more prevalent and evident in older groups. Concomitantly, the Müller cell processes which form the matrix between the photoreceptor nuclei appeared thicker. Similar find- 
ings were observed by Rodriguez-Muela et al. [30] who stated that the signs of neurodegeneration, including increased cell death in the retinal photoreceptor nuclei, start from 12 months of age onwards.

The present work demonstrated that the outer nuclear layer thickness decreased significantly in group II compared with group I and showed further significant decrease in group III. However, the senile group presented mild increase in the ONL thickness compared with group III, although the difference was statistically non-significant. This increase in thickness may be explained by the loosened structure that may occur in senile retinae Szabadfi et al. [34].

The present work revealed age-dependent structural changes at the ribbon synapses of photoreceptor synaptic terminals especially in rod spherules. Electron microscopic examination revealed decrease in the density of synaptic ribbons. Swollen, floated and fragmented ribbons were also encountered. These changes were progressive with age. In addition, progressive decrease of synaptic vesicles in rod spherules were present in senile retinae. These findings were in accordance with Dorfman et al. [14] who observed that the initial decline of ribbon synapses and the loss of synaptic sites was not complete in the aging rats.

In the present work, synaptophysin was used to mark synapses. Immunohistochemical staining revealed significant decrease in arborisation between photoreceptor axons and dendrites of bipolar cells and horizontal cells. Not only the density of synapses decreased, but also the thickness of this layer. OPL thickness was halved in group IV compared with group I. Meanwhile, a statistically non-significant difference was calculated between the two adult groups. Samuel et al. [31] pointed out that the thickness of the retina decreases with age and such thinning significantly affected both synaptic layers as well as the INL.

The present study showed that the INL was thinned out and was formed only of two to three cellular rows in the aged and senile groups. The morphological findings was confirmed by the statistical analysis; the INL thickness in group III recorded $60 \%$ of its initial thickness in the adult group and fell down to about one third in group IV. These findings were in accordance with Aggarwal et al. [1] who reported that aging causes loss of retinal neurons, including rod photoreceptors and rod bipolar cells.

Ultrastructural changes of the INL cells, in the present work were in the form of cytoplasmic vacuolations which increased in severity with aging. Vacu- oles were merged in the senile group so that bipolar and amacrine cells appeared to have ill-defined cell boundaries. In this work, IPL presented decreased arborisation. Moreover, the synaptic terminals showed patchy affection in the 12 months group and were more severely affected appearing as empty figures in the 24 months and 30 months groups. Furthermore, Fernàndez-Sánchez et al. [16] found that at 16 months of age, connectivity between photoreceptors and horizontal and bipolar cell processes were lost over some areas. In the present work, the morphological findings were confirmed by immunohistochemical examination and statistical analysis. SYN immunostaining showed significant decrease in the trabecular meshwork of synapses in the inner plexiform layer. The decrease was progressive and almost steady among the studied age groups. The optical density of SYN in group IV was found to be $42 \%$ of its density in group I. This work concluded 33\% decrease in IPL thickness in the aged group that was magnified to $50 \%$ in the senile group. Nieves-Moreno et al. [29] estimated linear regression of IPL thickness with aging using optical coherence tomography on volunteers of different age groups.

The present work revealed ganglion cells displayed vacuolations at the 12 months group. The degenerative vacuoles became more severe and extensive together with disrupted cytoplasmic membrane based on electron microscopy observations in the 24 and 30 months groups. The nerve fibre bundles were apparently smaller in senile group. Inner limiting membrane affection, in the form of thickening and exudation, was only observed in 30 months group. A study by Nag and Wadhwa [28] documented that cystic mitochondria and cystoid bodies appear in ganglion cells and nerve fibres in the aging human retina.

An important finding in this study was the apparent decrease and the irregular distribution of ganglion cells over the GCL, detected on light microscopic examination of the aged and senile specimens. Statistical analysis elucidated a significant decrease in ganglion cell count in group II; while the decrease in the subsequent groups was found to be non-significant. Fernàndez-Sánchez et al. [16] stated that in rodents, age causes morphologic changes and/or loss of retinal ganglion cells. Several works reported retinal ganglion cells or axonal loss [7]; some reported degeneration but no loss [37]. Yet, other authors perceived no loss in the $\mathrm{GCL}$ in aged animals [26]. Although some works have described that the retina thins with age, Feng et al. [15] 
found that this thinning is not accompanied by a reduction of the retinal volume. In the present study, numerous dilated blood capillaries were encountered in the GCL and the two plexiform layers. These blood capillaries became more dilated and more congested in the old and senile groups. Nadal-Nicolás et al. [26] clarified that the compensatory changes occurring in the capillaries have a negative impact on neuronal function. A decrease in capillary stability and new vessels formation, culminate in vessel loss in response to 'physiological hypoxia' and likely further reduce the ability of the microvasculature to meet the metabolic needs of the parenchyma.

The present work revealed that retinal capillaries of different layers of the retina displayed major thickening and lamination of the basement membrane with age. This seemed to cause outward displacement of the pericyte somas. Pericytes processes were embedded within the thick basement membrane. The same results were obtained by Donato et al. [13] who suggested that the observed age-dependent broadening of peripheral retinal capillaries may reflect the thickening of the capillary basement membrane and/or an increase in capillary luminal diameter similar to that demonstrated in regions of the aging brain. In healthy humans, increased proinflammatory cytokines have been observed in the aging vascular endothelial cells [13].

The present study showed that the retinal capillaries exhibited thickening of the internal and external basement membranes. Pericytes presented cytoplasmic inclusions and long cytoplasmic processes that extended and embedded in the thickened basement membrane. These changes are found to increase progressively with age. In a study performed by Bianchi et al. [3] on human eyes of elderly patients, similar results were obtained with highlighting the external basal lamina thickening. Their findings assigned a primary role to endothelial dysfunction as a cause of basement membrane thickening, while retinal alterations were considered to be a secondary cause of either ischaemia or exudation. In addition, the present work found that the endothelial cells lining the blood capillaries were severely affected and displayed progressive degeneration with age. Marked proliferation of the capillaries endothelial basal laminae was encountered. This was explained by Bianchi et al. [3] who illustrated that endothelial tight junction integrity was disrupted by various inflammatory cytokines and leukocyte-endothelial interaction.
Glial fibrillary acidic protein immunostaining in our study, were dramatically increased in the MG. Astrocytes at the nerve fibre layer together with Müller cells processes presented strong positive reaction in the aged retinae. With aging, the reaction became stronger and more numerous and thicker. Müller cells processes appeared traversing the IPL extending between ILM and OLM. Martinez-De Luna et al. [25] elucidated that the MG play a role in the extensive structural changes resulting in Müller cell hypertrophy and glial scar formation. They also explained those finding as mammalian MG respond to retinal injury by age changes summarised as reactive gliosis. Müller cell gliosis is a complex response that involves changes in cell physiology, gene expression and morphology. A hallmark of gliosis is the upregulation of intermediate filament proteins in glial cells, including GFAP [12]. Dysfunction of glial cells in different pathologies of the retina has been linked to retinal swelling and blood-retinal barrier breakdown [21].

The present study provides a baseline to better understanding of the implication of aging in rat models of human pathologies that are closely related to senescence. Elucidating the morphological changes in retinal aging would facilitate the design of new strategies to prevent visual dysfunction associated with both the normal aging process and age-related retinal pathologies.

\section{CONCLUSIONS}

It could be concluded that rat retinae clearly undergo age-related morphological changes on both light microscopic and ultrastructural levels and assimilated with immunohistochemistry and statistical analysis. Such changes provide a cellular base for explanation of decreased vision in humans with aging other than reflection errors.

All retinal layers were affected by aging with variable degrees. The changes in the retinal pigment epithelium and photoreceptors were prominent. Some changes started as early as the middle-aged group, while others appeared in the aged groups. Moreover, the alterations presented in the aged group were progressed and aggravated in the senile group. Effect of aging was not only qualitative, but also quantitative as proven by the statistical analysis. This was applied on the retinal neuronal synapses as well as somas. 


\section{RECOMMENDATIONS}

It is recommended to do further studies with linking the morphological findings of this study to the functional alterations that occur with normal aging. It is also recommended to investigate genetic factors and concomitant ocular diseases that aggravate the histological alterations occurring during the aging process.

The results of this study are hopefully to be taken in consideration to build strategies to decelerate or reverse age-associated deterioration in ocular function to avoid the psychosocial consequences of visual impairment in elderly.

\section{REFERENCES}

1. Aggarwal P, Nag TC, Wadhwa S. Age-related decrease in rod bipolar cell density of the human retina: an immunohistochemical study. J Biosci. 2007; 32(2): 293-298, indexed in Pubmed: 17435321.

2. Bancroft JD, Gamble M. Immunohistochemical technique. In: Theory and practice of histological techniques, 6 th ed. Churchill Livingstone, London 2008: 433-472.

3. Bianchi E, Ripandelli G, Taurone S, et al. Age and diabetes related changes of the retinal capillaries: An ultrastructural and immunohistochemical study. Int J Immunopathol Pharmacol. 2016; 29(1): 40-53, doi: 10.1177/0394632015615592, indexed in Pubmed: 26604209.

4. Bonilha VL. Age and disease-related structural changes in the retinal pigment epithelium. Clin Ophthalmol. 2008; 2(2): 413-424, indexed in Pubmed: 19668732.

5. Boulton ME. Studying melanin and lipofuscin in RPE cell culture models. Exp Eye Res. 2014; 126: 61-67, doi: 10.1016/j.exer.2014.01.016, indexed in Pubmed: 25152361.

6. Boya P, Esteban-Martínez L, Serrano-Puebla A, et al. Autophagy in the eye: Development, degeneration, and aging. Prog Retin Eye Res. 2016; 55: 206-245, doi: 10.1016/j. preteyeres.2016.08.001, indexed in Pubmed: 27566190.

7. Calkins DJ. Age-related changes in the visual pathways: blame it on the axon. Invest Ophthalmol Vis Sci. 2013; 54(14): ORSF37-ORSF41, doi: 10.1167/iovs.13-12784, indexed in Pubmed: 24335066.

8. Cattoretti G, Pileri S, Parravicini C, et al. Antigen unmasking on formalin-fixed, paraffin-embedded tissue sections. J Pathol. 1993; 171(2): 83-98, doi: 10.1002/ path.1711710205, indexed in Pubmed: 7506771.

9. Chernoivanenko IS, Matveeva EA, Gelfand VI, et al. Mitochondrial membrane potential is regulated by vimentin intermediate filaments. FASEB J. 2015; 29(3): 820-827, doi: 10.1096/fj.14-259903, indexed in Pubmed: 25404709.

10. Chirco KR, Sohn EH, Stone EM, et al. Structural and molecular changes in the aging choroid: implications for age-related macular degeneration. Eye. 2017; 31(1): 10-25, doi: 10.1038/eye.2016.216, indexed in Pubmed: 27716746.
11. Dan C, Jian-Bin T, Hui W, et al. Synaptophysin expression in rat retina following acute high intraocular pressure. Acta Histochem Cytochem. 2008; 41(6): 173-178, doi: 10.1267/ ahc.08034, indexed in Pubmed: 19180202.

12. de Pablo $Y$, Nilsson M, Pekna M, et al. Intermediate filaments are important for astrocyte response to oxidative stress induced by oxygen-glucose deprivation and reperfusion. Histochem Cell Biol. 2013; 140(1): 81-91, doi: 10.1007/s00418-013-1110-0, indexed in Pubmed: 23756782.

13. Donato $A J$, Black $A D$, Jablonski $K L$, et al. Aging is associated with greater nuclear NF kappa B, reduced I kappa $B$ alpha, and increased expression of proinflammatory cytokines in vascular endothelial cells of healthy humans. Aging Cell. 2008; 7(6): 805-812, doi: 10.1111/j.14749726.2008.00438.x, indexed in Pubmed: 18782346.

14. Dorfman AL, Cuenca N, Pinilla I, et al. Immunohistochemical evidence of synaptic retraction, cytoarchitectural remodeling, and cell death in the inner retina of the rat model of oygen-induced retinopathy (OIR). Invest Ophthalmol Vis Sci. 2011; 52(3): 1693-1708, doi: 10.1167/ iovs.10-6197, indexed in Pubmed: 21071736.

15. Feng L, Sun Z, Han H, et al. No age-related cell loss in three retinal nuclear layers of the Long-Evans rat. Vis Neurosci. 2007; 24(6): 799-803, doi: 10.1017/S0952523807070721, indexed in Pubmed: 18093367.

16. Fernández-Sánchez L, de Sevilla Müller LP, Brecha NC, et al. Loss of outer retinal neurons and circuitry alterations in the DBA/2J mouse. Invest Ophthalmol Vis Sci. 2014; 55(9): 6059-6072, doi: 10.1167/iovs.14-14421, indexed in Pubmed: 25118265.

17. Hippert $C$, Graca AB, Barber AC, et al. Müller glia activation in response to inherited retinal degeneration is highly varied and disease-specific. PLoS One. 2015; 10(3): e0120415, doi: 10.1371/journal.pone.0120415, indexed in Pubmed: 25793273.

18. Joly S, Pernet V, Samardzija M, et al. Pax6-positive Müller glia cells express cell cycle markers but do not proliferate after photoreceptor injury in the mouse retina. Glia. 2011; 59(7): 1033-1046, doi: 10.1002/glia.21174, indexed in Pubmed: 21500284.

19. Karthaus M, Falkenstein M. Functional Changes and Driving Performance in Older Drivers: Assessment and Interventions. Geriatrics. 2016; 1(2): 12, doi: 10.3390/ geriatrics1020012.

20. Keeling E, Lotery AJ, Tumbarello DA, et al. Impaired cargo clearance in the retinal pigment epithelium (RPE) underlies irreversible blinding diseases. Cells. 2018; 7(2), doi: 10.3390/cells7020016, indexed in Pubmed: 29473871.

21. Klaassen I, Van Noorden CJF, Schlingemann RO. Molecular basis of the inner blood-retinal barrier and its breakdown in diabetic macular edema and other pathological conditions. Prog Retin Eye Res. 2013; 34: 19-48, doi: 10.1016/j. preteyeres.2013.02.001, indexed in Pubmed: 23416119.

22. Litts KM, Messinger JD, Freund KB, et al. Inner segment remodeling and mitochondrial translocation in cone photoreceptors in age-related macular degeneration with outer retinal tubulation. Invest Ophthalmol Vis Sci. 2015; 
56(4): 2243-2253, doi: 10.1167/iovs.14-15838, indexed in Pubmed: 25758815.

23. Luke MPS, LeVatte TL, O'Reilly AM, et al. Effect of NCAM on aged-related deterioration in vision. Neurobiol Aging. 2016; 41: 93-106, doi: 10.1016/j.neurobiolaging.2016.02.003, indexed in Pubmed: 27103522.

24. Ma W, Coon S, Zhao L, et al. A2E accumulation influences retinal microglial activation and complement regulation. Neurobiol Aging. 2013; 34(3): 943-960, doi: 10.1016/j.neurobiolaging.2012.06.010, indexed in Pubmed: 22819137.

25. Martinez-De Luna RI, Ku RY, Aruck AM, et al. Müller glia reactivity follows retinal injury despite the absence of the glial fibrillary acidic protein gene in Xenopus. Dev Biol. 2017; 426(2): 219-235, doi: 10.1016/j.ydbio.2016.03.005, indexed in Pubmed: 26996101.

26. Nadal-Nicolás FM, Sobrado-Calvo $P$, Jiménez-López $M$, et al. Long-Term Effect of Optic Nerve Axotomy on the Retinal Ganglion Cell Layer. Invest Ophthalmol Vis Sci. 2015; 56(10): 6095-6112, doi: 10.1167/iovs.15-17195, indexed in Pubmed: 26393669.

27. Nadal-Nicolás FM, Vidal-Sanz M, Agudo-Barriuso M. The aging rat retina: from function to anatomy. Neurobiol Aging. 2018; 61: 146-168, doi: 10.1016/j.neurobiolaging.2017.09.021, indexed in Pubmed: 29080498.

28. Nag T, Wadhwa S. Ultrastructure of the human retina in aging and various pathological states. Micron. 2012; 43(7): 759-781, doi: 10.1016/j.micron.2012.01.011.

29. Nieves-Moreno M, Martínez-de-la-Casa JM, MoralesFernández L, et al. Impacts of age and sex on retinal layer thicknesses measured by spectral domain optical coherence tomography with Spectralis. PLoS One. 2018; 13(3): e0194169, doi: 10.1371/journal.pone.0194169, indexed in Pubmed: 29522565.

30. Rodríguez-Muela N, Koga H, García-Ledo L, et al. Balance between autophagic pathways preserves retinal homeo- stasis. Aging Cell. 2013; 12(3): 478-488, doi: 10.1111/ acel.12072, indexed in Pubmed: 23521856.

31. Samuel MA, Zhang $Y$, Meister $M$, et al. Age-related alterations in neurons of the mouse retina. J Neurosci. 2011; 31(44): 16033-16044, doi: 10.1523/JNEUROSCI.3580-11.2011, indexed in Pubmed: 22049445.

32. Sanes JR, Zipursky SL. Design principles of insect and vertebrate visual systems. Neuron. 2010; 66(1): 15-36, doi: 10.1016/j. neuron.2010.01.018, indexed in Pubmed: 20399726.

33. Sengupta $P$. The laboratory rat: relating its age with human's. Int J Prev Med. 2013; 4(6): 624-630, indexed in Pubmed: 23930179.

34. Szabadfi K, Estrada C, Fernandez-Villalba E, et al. Retinal aging in the diurnal Chilean rodent (Octodon degus): histological, ultrastructural and neurochemical alterations of the vertical information processing pathway. Front Cell Neurosc. 2015; 9, doi: 10.3389/fncel.2015.00126.

35. Warburton S, Davis WE, Southwick K, et al. Proteomic and phototoxic characterization of melanolipofuscin: correlation to disease and model for its origin. Mol Vis. 2007; 13 : 318-329, indexed in Pubmed: 17392682.

36. Yao J, Jia L, Shelby SJ, et al. Circadian and noncircadian modulation of autophagy in photoreceptors and retinal pigment epithelium. Invest Ophthalmol Vis Sci. 2014; 55(5): 3237-3246, doi: 10.1167/iovs.13-13336, indexed in Pubmed: 24781939.

37. Yassa HD. Age-related changes in the optic nerve of Sprague-Dawley rats: an ultrastructural and immunohistochemical study. Acta Histochem. 2014; 116(6): 1085-1095, doi: 10.1016/j.acthis.2014.05.001, indexed in Pubmed: 24958340.

38. Zhao JJ, Ouyang $\mathrm{H}$, Luo J, et al. Induction of retinal progenitors and neurons from mammalian Müller glia under defined conditions. J Biol Chem. 2014; 289(17): 11945-11951, doi: 10.1074/jbc.M113.532671, indexed in Pubmed: 24523410. 